\title{
An enhanced differential evolution algorithm with adaptation of switching crossover strategy for continuous optimization
}

\author{
Pikul Puphasuk, Jeerayut Wetweerapong *
}

\begin{abstract}
Designing an efficient optimization method which also has a simple structure is generally required by users for its applications to a wide range of practical problems. In this research, an enhanced differential evolution algorithm with adaptation of switching crossover strategy (DEASC) is proposed as a generalpurpose population-based optimization method for continuous optimization problems. DEASC extends the solving ability of a basic differential evolution algorithm (DE) whose performance significantly depends on user selection of the control parameters: scaling factor, crossover rate and population size. Like the original DE, the proposed method is aimed at efficiency, simplicity and robustness. The appropriate population size is selected to work in accordance with good choices of the scaling factors. Then, the switching crossover strategy of using low or high crossover rates are incorporated and adapted to suit the problem being solved. In this manner, the adaptation strategy is just a convenient add-on mechanism. To verify the performance of DEASC, it is tested on several benchmark problems of various types and difficulties, and compared with some well-known methods in the literature. It is also applied to solve some practical systems of nonlinear equations. Despite its much simpler algorithmic structure, the experimental results show that DEASC greatly enhances the basic DE. It is able to solve all the test problems with fast convergence speed and overall outperforms the compared methods which have more complicated structures. In addition, DEASC also shows promising results on high dimensional test functions.
\end{abstract}

Keywords: Continuous optimization, enhanced differential evolution algorithm, control parameter adaptation, switching crossover strategy

*Deparment of Mathematics, Faculty of Science, Khon Kaen University, Khon Kaen, 40002, Thailand,ppikul@kku.ac.th,wjeera@kku.ac.th 


\section{Introduction}

Finding the optimal solutions to complex computational problems in engineering and applied sciences is an important and challenging task which often requires welldesigned efficient methods. In the past few decades, numerous computational intelligence methods have been devised to overcome the nonlinearity, nonseparability and multimodality of decision variables in the objective functions of the problems. Among them, the evolutionary methods which include the genetic algorithm (GA), the particle swarm optimization (PSO), the differential evolution algorithm (DE) and other nature-inspired optimization methods have been widely studied and applied to solve practical problems in various fields $[2,8,17,27,29]$. Many modified or enhanced variants have been continuously proposed $[1,5,18]$.

In this research, we focus on enhancing the performance of the basic DE algorithm which is a well-known population-based optimization method for continuous optimization problems, proposed by Storn and Price during the years 1995-1997 [25,26]. DE has a simple structure with few control parameters and can be made efficient for different types of problems through suitable selection of the control parameters. However, there exist no general rules of recommendations for users and it is well recognized that DE's performance is sensitive to its three control parameters: scaling factor, crossover rate and population size. First, the appropriate population size must be chosen, which generally depends on the dimension and difficulty of the problem. Small populations can easily lose diversity during the evolutionary process and lead to stagnant or premature convergence while large populations require more computations. The scaling factor is known to affect the step sizes of mutant vectors and the acceleration of the convergence to a local or an optimal solution. Similar to the population size, too small scaling factor values will lead to premature convergence or stagnation while too large values can cause a very low speed of convergence. The crossover rate, on the other hand, plays an important role in a different manner and needs more attention. Its good values are roughly known to associate to the types of the objective functions. What is more crucial and rarely known is how these three control parameters affect each other. This explains why so many enhanced DE variants that improve the basic $\mathrm{DE}$ in different aspects are designed, proposed and tested for different problems.

Our proposed DEASC algorithm is designed to be an effective DE variant with the simplest enhanced structures as possible. It uses the simple range of the scaling factor values to promote the diversity of mutant vectors while still keeping the good convergence performance. This enhancement also reduces the population size problem by allowing the effective use of smaller population size. Another equally important enhancement is the simple adaptation of using the mixture of the low and high values of $C R$ in a switching manner, which enables DEASC to adapt to the problem being solved.

The remainder of the paper is organized as follows. The next Section 2 presents the literature review concerning the basic DE algorithm, previous recommendation rules for the control parameters, and the enhanced DE variants. The proposed differential evolution algorithm with adaptation of switching crossover strategy (DEASC) is described in Section 3. Five experiments concerning the design of DEASC and its 
performances are conducted, and the results and discussion are given in Section 4 . Finally, the conclusions are made in the last section.

\section{Literature review}

To facilitate understanding the review of related literature, the concepts and structure of the basic DE algorithm are given first. Then the previous recommendation rules for its control parameters are presented and some enhanced DE variants are discussed.

\subsection{The basic DE algorithm}

Differential evolution algorithm $[25,26]$ is a stochastic population-based optimization similar to genetic algorithm and evolutionary algorithm. It consists of three common basic evolutionary operations: mutation, crossover (or recombination) and selection. After the initialization of the $N P$ population vectors, a mutant vector $x m$ is generated for each target vector $x_{i}$ by adding the scaled difference of two random vectors to the other random one: $x m=x_{r 1}+F\left(x_{r 2}-x_{r 3}\right)$, where $F$ is the scaling factor. The mutant vector is crossovered with the target to generate a trial vector by using the binomial crossover with the crossover rate $C R$. Then, the selection replaces the target vector with the trial vector if it is fitter. This iterative evolutionary process is repeated until reaching the specified stopping condition. The steps of the basic DE algorithm are as follows .

Step 1 Inputs: Objective function to be minimized $(f)$, problem dimension $(D)$, and population size $(N P)$.

Step 2 Initialization: Randomly initialize all vectors of the population matrix $P=$ $\left[x_{i j}\right]$ of size $N P \times D$ with real values between the lower and upper bounds $L B$ and $U B$. Then, calculate the fitness for each population vector and record the best vector $x$ best and the best value fbest.

Step 3 Mutation: For each target population vector $x_{i}$, construct the mutant vector $x m$ by

$$
x m=x_{r 1}+F\left(x_{r 2}-x_{r 3}\right)
$$

where $r 1, r 2$ and $r 3$ are randomly generated distinct indices (in the range of 1 to $N P$ ) which are also different from the target index $i$, and $F$ is a scaling factor.

Step 4 Crossover: Construct the trial vector $x c$ by replacing some components of $x_{i}$ with the corresponding components of $x m$ as follows:

$$
x c_{j}= \begin{cases}x m_{j} & ; \text { rand }_{j}<C R \text { or } j=I C, \\ x_{i j} & ; \text { otherwise }\end{cases}
$$

where $\operatorname{rand}_{j}$ is a uniform random number in $[0,1], C R$ is a crossover rate, and $I C$ is a randomly fixed index from 1 to $D$. 
Step 5 Selection: Apply the greedy selection. If $f(x c)<f\left(x_{i}\right)$, then replace the target vector $x_{i}$ with $x c$. If $f(x c)<f$ best, then update $x b e s t$ with $x c$ and update fbest with $f(x c)$.

\section{Step 6 Stopping condition:}

Repeat all the steps (3) - (5) until fbest is less than the value to reach $(V T R)$ or the maximum number of function evaluations $(\operatorname{maxn} f)$ is reached. Then report the obtained best solution.

\subsection{Previous recommendation rules for the control parameters of the basic DE}

In 1997, the DE algorithm has been introduced and shown to outperform many other optimization methods available at that time [26]. The authors have chosen several combinations of the population sizes, scaling factor values and crossover rates $(N P, F, C R)$ for minimizing different test functions with different dimensions. They have claimed that DEs control variables, $N P, F$ and $C R$, are not difficult to choose in order to obtain good results, and suggested that the population sizes should be between $5 D$ to $10 D$. Also note that in their experiments the different values to reach (precisions) are set for different test functions i.e., the lower precisions are used for the harder problems.

In 2000, Lampinen and Zelinka have experimented on the situation that the DE algorithm has lost its capability of finding better solutions for the current evolutionary optimization process, which is called the stagnation [10]. To avoid this problem, the number of potential trial solutions should be increased by using a larger population size, and the $F$ and $C R$ values which are not equal to 1.0. Such values will reduce the number of potential trial solutions dramatically when compared to those which are less than 1.0. The stagnation is different from premature convergence. The former case can happen while the population is still diverse whereas in the later case, the population vectors converge (become the same point) to a local minimum point or a non-local minimum point in the search space.

Later in 2002, Gamperle et al. have shown that the application of DE on several test functions for finding the global optimum is sensitive to the choice of the control parameters [7]. Although empirical rules are provided in the literature [26], choosing the proper parameters for DE is more difficult than expected. The scaling factor $F$ should not be smaller than a certain value to prevent premature convergence and this value depends on the test function and on the other control parameters. The larger $C R$ can speed up the convergence but from a certain value upwards the convergence speed may decrease or the population may converge prematurely. They have suggested that the population size $N P$ should be between $3 D$ to $8 D$.

In 2005, Ronkkonen et al. have entered the classic DE to the CEC2005 contest to perform on the 25 scalable test functions at the dimension $D=10$ and 30 [23]. The test functions include many nonseparable and highly multimodal functions. The maximum number of function evaluations is set at $10000 D$ and they have chosen the setting of $N P=30, F=0.9$, and $C R=0.9$ for all test functions at both two 
dimensions. The basic DE with this setting can solve almost all the test functions at $D=10$ giving the solutions at the value to reach $V T R=10^{-8}$ while it fails to solve almost all the test functions at $D=30$ due to the allowed maximum number of function evaluations which is too low. Their results clearly show that the good settings of DE depend on the dimensions and the difficulties of the problems being solved, and the allowed function evaluations also affect the DE's performance.

Also in 2005, Das et al. have presented two improved variants of the classic DE: DE with random scaling factor (DERSF) in the range of $[0.5,1]$, and DE with time varying scaling factor (DETVSF) where its value is linearly reduced from 1.2 to 0.4 [4]. These two DE variants are performed on seven test functions at $D=10,20$ and 30 , and the population size is chosen to be $10 D$. Different number of maximum generations allowed and the $V T R$ values are set for different functions (due to their difficulties). The results show that both two DE variants can statistically improve the performance of the basic DE. However, they can solve only the easy Sphere function but cannot solve all the other test functions successfully for all 50 runs. They have succeeded less than $50 \%$ of the 50 runs for the Rosenbrock function at $D=20$ and 30 .

Zielinski et al. in 2006 have studied the control parameters of DE using the power allocation problems [31]. The parallel interference cancellation (PIC) optimization problem is used for studying the effect of different parameter settings. The DE control parameters $F$ and $C R$ are varied from 0.1 to 1.0 in steps of 0.1 , respectively and 100 independent runs are performed for every parameter combination. The dimension $D$ is 16 and the population size $N P=5 D=80$ is used. It is found that convergence is always reached for $C R \geq 0.6$ and $F \geq 0.6$, and convergence is never obtained for small values of $C R \leq 0.2$ or $F \leq 0.2$. For this problem, $F=0.7, C R=0.9$ are recommended. Then with this setting for $F$ and $C R$, the dependence on $N P$ is examined. The number of individuals is varied in the range $10 \leq N P \leq 100$ with a step size of 10 . The results show that the surprisingly low number of 30 individuals is sufficient for a reliable convergence behavior.

Also in 2006, Mezura et al. have presented an empirical comparison of some DE variants to solve global optimization problems [15]. They have aimed to identify a suitable variant depending on the problem's feature and the best one with the best performance regardless of the features of the problem to be solved. Eight variants are tested on 13 benchmark problems at the fixed dimension $D=30$. The setting of $N P=60, V T R=10^{-12}$, and a maximum number of generations equal to 2000 is used. The $F$ and $C R$ values are varied from 0.3 to 0.9 , and 0.1 to 0.9 in steps of 0.1 , respectively. For all approaches, the $F$ value is generated anew in such range at each generation while the $C R$ value is tuned for each problem. They have noted that the $C R$ values close to the extreme value $C R \in[0.8,0.9]$ or $[0.0,0.3]$ are the most common, which suggests that the influence of the parent (the target vector) should be either significant or very small.

In 2008, Mallipeddi and Suganthan have investigated the effect of population size on the quality of solutions and the computational effort required by DE algorithm [13]. Five problems chosen from the CEC2005 problem set are used at $D=10$ and 30 . The fixed values of $F=0.5$ and $C R=0.3$ are used with the different population sizes 
$N P=2 D, 4 D, 6 D$, and $8 D$. The maximum number of function evaluations is set to 100,000 and 30,0000 for the 10-dimensional and the 30-dimensional problems, respectively, with the $V T R=10^{-5}$. It is found that a smaller population with a greedy strategy converges fast but the effect of premature convergence and stagnation is more prominent. In contrast, a large population with a strategy having good exploration capacity reduces the probability of premature convergence and stagnation effects, but the convergence speed can be slower.

Storn in 2008 has presented a review of trends and open questions for DE research [24]. One important research direction is basic DE research which investigates inner workings and theoretical aspects of DE with the goal of better understanding DE, identifying its weaknesses and improving its performances. For a decade, tuning and finding suitable three main control variables $N P, F$, and $C R$, and their bounds has been a topic of intensive research. Referring to [26] and [23], the following control values are suggested: $F$ in $[0.5,1.0], C R$ in $[0.8,1.0]$ and $N P=10 D$. High values of $C R$ are recommended for promoting transfer of points between two basins of attraction and for rotationally invariant moves while low values of $C R$ are advantageous for separable functions by concentrating the search on the axes of the coordinate system. Since the recommended values still lack generality [7], finding the best settings of $F, C R$, and $N P$ automatically has become one of the research trends [3,15]. It is observed that many proposed adaptive DE variants are compared by using only a fixed number of evaluations and thus no behaviors for the long runs are reported. The author has strongly suggested the investigation of performance comparisons by using fixed values of precision (value to reach).

\subsection{Enhanced DE variants}

In this subsection, we review some selected well-known enhanced DE variants which include the adaptive and self-adaptive DE algorithms. Some of them will be used to compare their performances with that of the proposed DEASC.

In 2006, Brest et al. have proposed a self-adaptive DE algorithm called jDE in which each individual population vector is extended with its own parameters $F$ and $C R$ [3]. Before mutation and crossover operations are performed, the new values are generated in the ranges of $[0.1,0.9]$ and $[0,1.0]$, respectively, with the probabilities equal to 0.1. Otherwise, the same values presented in the target vector (parent) are used. Then, the selection process propagates the good parameter values to the next generation. The jDE is tested on several benchmark functions and shown to outperform the basic DE with $F=0.5$ and $C R=0.9$.

Qin and Suganthan in 2009 have presented an adaptive DE called SaDE [22]. Four mutation strategies are used with equal initial probabilities. The probability for each strategy is adjusted according to its success counts in the selection process. Before mutation and crossover operations are performed, the parameters $F_{j}$ and $C R_{j}$, associated to the chosen strategy, are generated by normal distributions using the associated centers. These centers are dynamically updated for every fixed learning period of generations. The $\mathrm{SaDE}$ is shown to outperform overall the jDE and the 
basic DE algorithms with various static values of $F$ and $C R$.

Also in 2009, Zhang and Sanderson have introduced an adaptive DE with an external archive called JADE [30]. The algorithm implements a new mutation strategy that utilizes some top best individuals and the archive operation that utilizes historical data. Before mutation and crossover operations are performed, the parameters $F$ and $C R$ are generated by Cauchy and normal distributions, respectively. Their associated centers are updated at the end of each generation according to the extracted information obtained from the sets of successful values. JADE is shown to perform better than jDE, SaDE and the basic DE with $F=0.5$ and $C R=0.9$.

In 2016, Leon and Xiang have presented a simple adaptive DE called GADE which adds the greedy adjustment of the control parameters $F$ and $C R$ [11]. The initial centers of $F$ and $C R$ are set to 0.5 . Then their two neighborhood values $F-d 1$ and $F+d 1$, and $C R-d 2$ and $C R+d 2$, with $d 1=d 2=0.1$, are generated and used in mutation and crossover operations. The best values are identified during the learning period and used to update the new centers. GADE is shown to overall outperform the basic DE with $F=0.9$ and $C R=0.9$, SaDE and JADE.

There are many enhanced DE variants proposed in the literature. Rawaa et al. have reviewed the algorithmic design issues in adaptive differential evolution schemes in 2018 [1]. From their classification illustration, jDE and GADE are adaptive DE algorithms with a single DE strategy and a standard mutation strategy while JADE uses an advanced mutation strategy, and SaDE uses multiple DE strategies with a standard mutation strategy. These 4 variants are among the well-known adaptive DE algorithms.

Recently in 2019, Li et al. have proposed a self-feedback DE called SFDE [12]. The algorithm calculates the indicator number to approximate the number of optima in the fitness landscape presented by the population points and uses this number to adapt to the fitness landscape characteristics of the problem. In SFDE, two mutation strategies are used according to the low values or high values of the indicator numbers, respectively. It also incorporates other techniques to enhance its performance: the normal and Cauchy distributions to sample the $F$ and $C R$ values from their centers and the selection strategy which uses the best and worst individuals for the next generation. Using the suitable $F$ values (centers) for each test function, which are obtained from the preliminary experiment, SFDE is shown to outperform the basic DE with $F=0.5$ and $C R=0.9$.

\section{Differential evolution algorithm with adaptation of switch- ing crossover strategy (DEASC)}

The key principle for optimizing a function is how to balance the local search (intensifying) and global search (diversifying), and adapt the search at different stages of the optimization process. DEASC encounters this by using the mixture of the low and high values of $C R$ in a switching manner, which enables DEASC to adapt to the problem being solved. The low crossover rate $C R \in[0,0.1]$ corresponds to a local search while the high crossover rate $C R \in[0.9,1.0]$ corresponds to a global search. 
The local search explores and creates several good candidate solutions in different regions of the search domain. Then the global search combines and exchanges between those good solutions. DEASC also uses the simple range of the scaling factor values to promote the diversity of mutant vectors while still keeping the good convergence performance. This enhancement reduces the population size problem by allowing the effective use of smaller population size. The DEASC algorithm can be described as follows.

\section{Inputs and control parameters:}

Objective function to be minimized : $f$

Problem dimension: $D$

Lower and upper bounds of the problem: $L B, U B$

Population size: $N P=40$

Value to reach: $V T R=10^{-10}$

Maximum number of function evaluations: $\operatorname{maxnf}$

Scaling factor: $F$ in the range of $[0.5,0.7]$

Crossover rate: $C R$ in the range of $[0,0.1]$ or $[0.9,1]$

The initial probabilities for using low or high crossover rates: $p c 1=p c 2=0.5$

The initial counters corresponding to $p c 1$ and $p c 2: n c 1=n c 2=0$

\section{Initialization:}

Randomly initialize all vectors of the population matrix $P=\left[x_{i j}\right]$ of size $N P \times D$ with real values between the lower and upper bounds $L B$ and $U B$. Calculate the fitness (the objective function value) for each population vector and record the best vector $x$ best and the best value fbest.

\section{Mutation:}

For each target population vector $x_{i}$, randomly generate distinct indices $r 1, r 2$ and $r 3$ (in the range of 1 to $N P$ ) which are also different from the target index $i$. Construct the mutant vector $x m$ by

$$
x m=x_{r 1}+F\left(x_{r 2}-x_{r 3}\right)
$$

where $F$ is randomly generated in $[0.5,0.7]$.

\section{Crossover:}

Generate a uniform random number $u$ in $[0,1]$. If $u<p c 1$, then random a crossover rate $C R$ in $[0,0.1]$; otherwise, random $C R$ in $[0.9,1]$. Construct the trial vector $x c$ by replacing some components of $x_{i}$ with the corresponding components of $x m$ as follows:

$$
x c_{j}= \begin{cases}x m_{j} & ; \text { rand }_{j}<C R \text { or } j=I C, \\ x_{i j} & ; \text { otherwise }\end{cases}
$$

where $\operatorname{rand}_{j}$ is a uniform random number in $[0,1]$ and $I C$ is a randomly fixed index from 1 to $D$ for this crossover.

\section{Selection:}

Apply the greedy selection and check for an update of xbest. If $f(x c)<f\left(x_{i}\right)$, 
then replace the target vector $x_{i}$ with $x c$. If $f(x c)<f b e s t$, then update $x b e s t$ with $x c$ and update fbest with $f(x c)$.

\section{Updating control parameters:}

Update $p c_{1}$ and $p c_{2}$ as follows.

(6.1) If a better solution found in the selection is generated with $C R \in[0,0.1]$, then increase $n c_{1}:=n c_{1}+1$; otherwise, increase $n c_{2}:=n c_{2}+1$.

(6.2) If $n c_{1}+n c_{2} \geq 100$, then adjust the counters $n c_{1}:=n c_{1}+10$ and $n c_{2}:=$ $n c_{2}+10$ to prevent both of them from 0 .

(6.3) Update the probabilities using the weighted formula $p c_{1}=0.9 p c_{1}+0.1\left(n c_{1}\right) /(n c 1+n c 2)$ and $p c_{2}=1.0-p c_{1}$.

(6.4) Reset the counters $n p c 1=0, n p c 2=0$ when the probabilities $p c 1$ and $p c 2$ are updated.

\section{Stopping condition:}

Repeat all the steps (3) - (6) until fbest is less than $V T R$ or maxnf is reached. Then report the obtained best solution.

The flowchart of DEASC is shown in Figure 1.

\section{Preliminary experiments and results}

This section presents two preliminary experiments. They are designed for constructing the proposed DEASC algorithm. The first experiment concerns the effect of using low and high crossover rates and the second one investigates the strategies for mixing them.

\subsection{Test functions}

The test functions used in this study are listed in Table 1. They consist of 8 benchmark functions of 4 different types: unimodal and separable (US), unimodal and nonseparable (UN), multimodal and separable (MS), and multimodal and nonseparable (MN). Their formulations, types, global optima, and search ranges are given. For the preliminary experiments, four representative functions are selected.

\subsection{Effect of low and high crossover rates on the performance of basic DE}

In this experiment, the effect of the crossover rates $C R=0.1$ and $C R=0.9$ to the performance of basic DE are studied. The crossover rate $C R=0.9$ and the scaling factor $F=0.5$ are generally recommended in the literature [24]. We aim to show that 


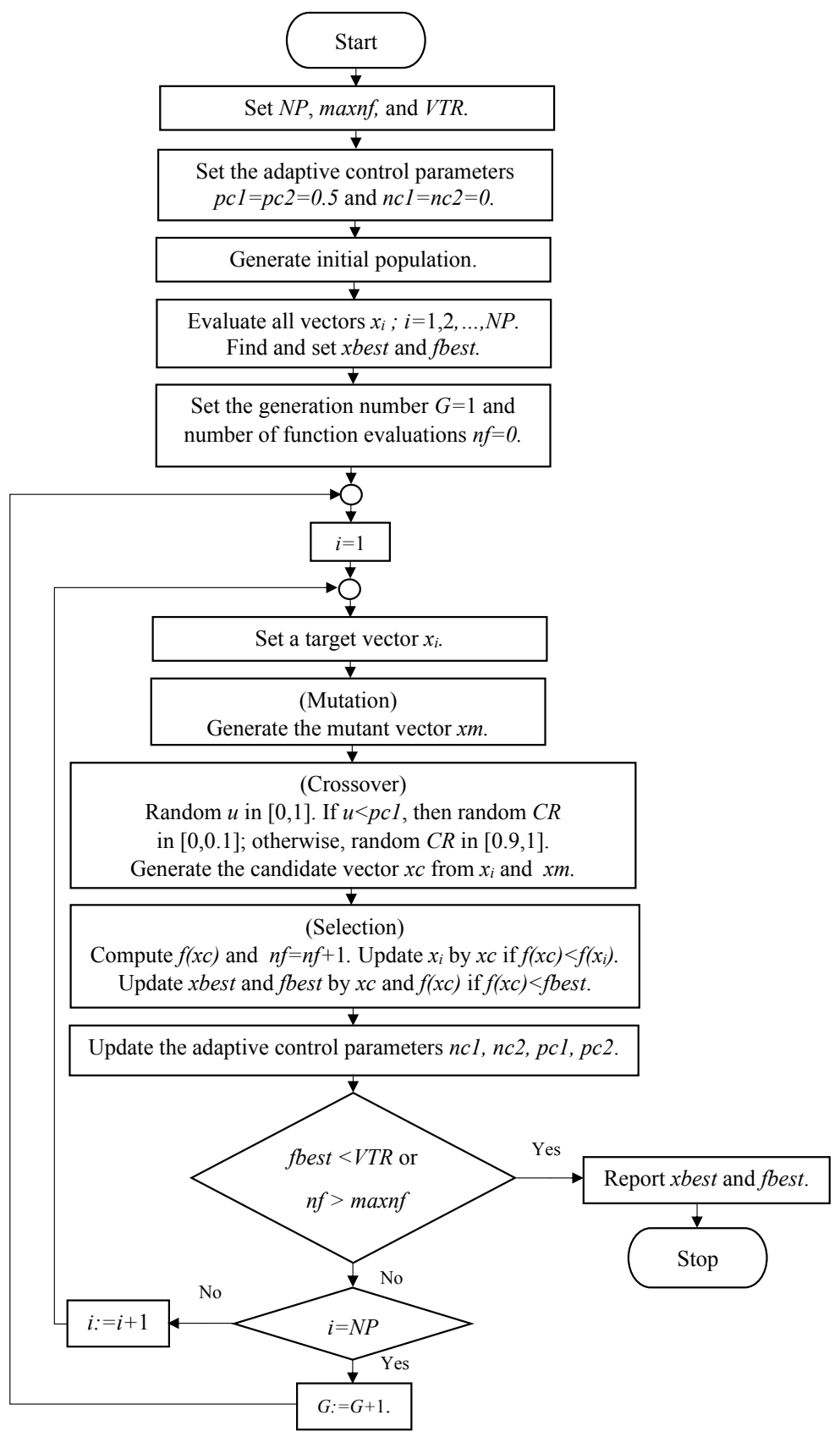

Figure 1: Flowchart of the proposed DEASC algorithm. 
Table 1: Test functions $[11,30]$.

\begin{tabular}{|c|c|c|c|c|}
\hline Function & Formulation & Type & $\begin{array}{c}\text { Global } \\
\text { optimum }\end{array}$ & $\begin{array}{c}\text { Search } \\
\text { range }\end{array}$ \\
\hline Sphere & $f(\mathbf{x})=\sum x_{i}^{2}$ & US & $\overline{0}$ & {$[-100,100]^{D}$} \\
\hline Schwefel 1.2 & $f(\mathbf{x})=\sum\left(\sum x_{j}\right)$ & US & $\overline{0}$ & {$[-100,100]^{D}$} \\
\hline Rosenbrock & $f(\mathbf{x})=\sum\left[100\left(x_{i+1}-x_{i}^{2}\right)^{2}+\left(x_{i}-1\right)^{2}\right]$ & UN & $\overline{1}$ & {$[-100,100]^{D}$} \\
\hline Schwefel 2.22 & $f(\mathbf{x})=$ & UN & $\overline{0}$ & {$[-10,10]^{D}$} \\
\hline Rastrigin & $f(\mathbf{x})=10 D+\sum\left[x_{i}^{2}-10 \cos \left(2 \pi x_{i}\right)\right]$ & MS & $\overline{0}$ & {$[-5.12,5.12]^{D}$} \\
\hline Schwefel & $\begin{aligned} f(\mathbf{x})= & 418.98288727243369 D \\
& -\sum^{D} x_{i} \sin \sqrt{\left(\left|x_{i}\right|\right)}\end{aligned}$ & MS & $\overline{420.96}$ & {$[-500,500]^{D}$} \\
\hline Ackley & $f(\mathbf{x})=-20 \exp \left(-0.2 \sqrt{\frac{1}{D}} \sum^{D} x_{i}^{2}\right)$ & $\mathrm{MN}$ & $\overline{0}$ & {$[-32,32]^{D}$} \\
\hline Griewank & $f(\mathbf{x})=\sum_{i=1} \frac{x_{i}^{2}}{4000}-\prod_{i=1} \cos \left(\frac{x_{i}}{\sqrt{i}}\right)+1$ & $\mathrm{MN}$ & $\overline{0}$ & {$[-600,600]^{D}$} \\
\hline
\end{tabular}

$C R=0.1$ also gives good convergence performance for different types of functions. Two scaling factor values $F=0.5$ and $F=0.7$ are used where $F=0.7$ is aimed to prevent premature convergence and stagnation. Four representative functions $(2$ unimodal and 2 multimodal functions) are chosen to show these effects. The population sizes and dimensions are varied as $N P=40,60,80$ and $D=10,30$. The $V T R=10^{-10}$ and $\operatorname{maxnf}=40,000 D$ are used, and each configuration is performed 50 independent runs. The number of successful runs $(N S)$, the mean of number of function evaluations (Mean nf), and the percentage of standard deviations of the function evaluations (\%SD) are reported in Table 2 and Table 3.

In these tables, for each test function, the $C R$ values $(0.1,0.5$ or 0.9$)$ that show no serious convergence problems $(N S=0)$ with most combinations of $F$ value (0.5 or 0.7$)$ and $N P$ values $(40,60$, or 80$)$, and give the smallest numbers of function evaluations are highlighted in bold. Among these 18 combinations of $(F, C, N P)$, we can observe that either $C R=0.1$ or 0.9 appears to be a suitable choice for each function i.e, $C R=0.1$ is suitable for multimodal Rastrigin and Griewank functions while $C R=0.9$ 
Table 2: Performance of DE with low and high crossover rates averaged over 50 independent runs for Schwefel 1.2 and Rosenbrock functions.

\begin{tabular}{|c|c|c|c|c|c|c|c|}
\hline \multirow[t]{2}{*}{ Functions } & \multirow[t]{2}{*}{$C R$} & \multirow[t]{2}{*}{$F$} & \multirow[t]{2}{*}{$N P$} & \multicolumn{2}{|r|}{$D=10$} & \multicolumn{2}{|r|}{$D=30$} \\
\hline & & & & $N S$ & Mean $\mathrm{nf}(\% \mathrm{SD})$ & $N S$ & Mean $\mathrm{nf}(\% \mathrm{SD})$ \\
\hline \multirow[t]{18}{*}{ Schwefel 1.2} & \multirow[t]{6}{*}{0.1} & \multirow[t]{3}{*}{0.5} & 40 & 50 & $212359(3.59)$ & 0 & - \\
\hline & & & 60 & 50 & $319124(2.84)$ & 0 & - \\
\hline & & & 80 & 50 & $423305(3.17)$ & 0 & - \\
\hline & & \multirow[t]{3}{*}{0.7} & 40 & 50 & $276274(3.48)$ & 0 & - \\
\hline & & & 60 & 50 & $416475(2.66)$ & 0 & - \\
\hline & & & 80 & 50 & $558305(2.72)$ & 0 & - \\
\hline & \multirow[t]{6}{*}{0.5} & \multirow[t]{3}{*}{0.5} & 40 & 50 & $60145(3.66)$ & 0 & - \\
\hline & & & 60 & 50 & $94593(3.28)$ & 0 & - \\
\hline & & & 80 & 50 & $127539(2.63)$ & 0 & - \\
\hline & & \multirow[t]{3}{*}{0.7} & 40 & 50 & $115593(2.94)$ & 0 & - \\
\hline & & & 60 & 50 & $178343(2.89)$ & 0 & - \\
\hline & & & 80 & 50 & $240004(2.59)$ & 0 & - \\
\hline & \multirow[t]{6}{*}{0.9} & \multirow[t]{3}{*}{0.5} & 40 & 50 & $18173(12.86)$ & 50 & $215157(10.74)$ \\
\hline & & & 60 & 50 & $26559(3.31)$ & 50 & $251009(5.54)$ \\
\hline & & & 80 & 50 & $37794(2.87)$ & 50 & $338814(3.76)$ \\
\hline & & \multirow[t]{3}{*}{0.7} & 40 & 50 & $39109(3.36)$ & 50 & $410792(3.81)$ \\
\hline & & & 60 & 50 & $67865(2.92)$ & 50 & $910653(2.97)$ \\
\hline & & & 80 & 50 & $96238(2.58)$ & 50 & $1709011(2.98)$ \\
\hline \multirow[t]{18}{*}{ Rosenbrock } & \multirow[t]{6}{*}{0.1} & \multirow[t]{3}{*}{0.5} & 40 & 0 & - & 0 & - \\
\hline & & & 60 & 0 & - & 0 & - \\
\hline & & & 80 & 0 & - & 0 & - \\
\hline & & \multirow[t]{3}{*}{0.7} & 40 & 0 & - & 0 & - \\
\hline & & & 60 & 0 & - & 0 & - \\
\hline & & & 80 & 0 & - & 0 & - \\
\hline & \multirow[t]{6}{*}{0.5} & \multirow[t]{3}{*}{0.5} & 40 & 3 & 104251(15.92) & 0 & - \\
\hline & & & 60 & 0 & - & 0 & - \\
\hline & & & 80 & 2 & 287321(-) & 1 & 1130329(-) \\
\hline & & \multirow[t]{3}{*}{0.7} & 40 & 50 & $191010(3.59)$ & 0 & - \\
\hline & & & 60 & 50 & $288350(3.36)$ & 0 & - \\
\hline & & & 80 & 46 & $385252(2.49)$ & 0 & - \\
\hline & \multirow[t]{6}{*}{0.9} & \multirow[t]{3}{*}{0.5} & 40 & 3 & $382342(82.07)$ & 0 & - \\
\hline & & & 60 & 50 & $70929(28.21)$ & 45 & $764608(21.86)$ \\
\hline & & & 80 & 50 & $64029(7.40)$ & 50 & $371291(5.96)$ \\
\hline & & \multirow[t]{3}{*}{0.7} & 40 & 50 & $50188(4.73)$ & 49 & $363209(5.14)$ \\
\hline & & & 60 & 50 & $81248(3.28)$ & 50 & $674930(2.43)$ \\
\hline & & & 80 & 50 & $112236(2.64)$ & 50 & $1129129(2.53)$ \\
\hline
\end{tabular}

is suitable for unimodal and nonseparable Schwefel 1.2 and Rosenbrock functions. With the suitable $C R$ values, using $F=0.5$ gives good convergence results for almost cases except for Rosenbrock function with $N P=40$ where it gives $N S=0$ while using $F=0.7$ gives $N S=49$, and $F=0.7$ provides a convergence result for every case that $F=0.5$ provides a convergence result but $F=0.7$ requires more function evaluations. 
Table 3: Performance of DE with low and high crossover rates averaged over 50 independent runs for Rastrigin and Griewank functions.

\begin{tabular}{|c|c|c|c|c|c|c|c|}
\hline \multirow[t]{2}{*}{ Functions } & \multirow[t]{2}{*}{$C R$} & \multirow[t]{2}{*}{$F$} & \multirow[t]{2}{*}{$N P$} & \multicolumn{2}{|r|}{$D=10$} & \multicolumn{2}{|r|}{$D=30$} \\
\hline & & & & $N S$ & Mean $\mathrm{nf}(\% \mathrm{SD})$ & $N S$ & Mean $\mathrm{nf}(\% \mathrm{SD})$ \\
\hline \multirow[t]{18}{*}{ Rastrigin } & \multirow[t]{6}{*}{0.1} & \multirow[t]{3}{*}{0.5} & 40 & 50 & $16321(2.79)$ & 49 & $68693(2.33)$ \\
\hline & & & 60 & 50 & $24051(2.63)$ & 50 & $104034(2.74)$ \\
\hline & & & 80 & 50 & $32347(2.17)$ & 50 & $140095(1.96)$ \\
\hline & & \multirow[t]{3}{*}{0.7} & 40 & 49 & $20995(3.70)$ & 47 & $101060(3.65)$ \\
\hline & & & 60 & 50 & $31448(3.16)$ & 50 & $156912(3.21)$ \\
\hline & & & 80 & 50 & $41668(2.21)$ & 50 & $210868(3.36)$ \\
\hline & \multirow[t]{6}{*}{0.5} & \multirow[t]{3}{*}{0.5} & 40 & 50 & $38206(12.11)$ & 0 & - \\
\hline & & & 60 & 50 & $59692(6.98)$ & 0 & - \\
\hline & & & 80 & 50 & $82638(4.76)$ & 0 & - \\
\hline & & \multirow[t]{3}{*}{0.7} & 40 & 50 & $67719(13.53)$ & 0 & - \\
\hline & & & 60 & 50 & $107163(11.91)$ & 0 & - \\
\hline & & & 80 & 50 & $152518(10.40)$ & 0 & - \\
\hline & \multirow[t]{6}{*}{0.9} & \multirow[t]{3}{*}{0.5} & 40 & 13 & $44075(26.26)$ & 0 & - \\
\hline & & & 60 & 28 & 113796(23.81) & 0 & - \\
\hline & & & 80 & 40 & $205760(27.19)$ & 0 & - \\
\hline & & \multirow[t]{3}{*}{0.7} & 40 & 4 & 92922(18.29) & 0 & - \\
\hline & & & 60 & 7 & $200261(27.27)$ & 0 & - \\
\hline & & & 80 & 19 & $316725(30.99)$ & 0 & - \\
\hline \multirow[t]{18}{*}{ Griewank } & \multirow[t]{6}{*}{0.1} & \multirow[t]{3}{*}{0.5} & 40 & 50 & $27010(12.07)$ & 50 & $41272(4.83)$ \\
\hline & & & 60 & 50 & $39314(8.57)$ & 50 & $61458(3.13)$ \\
\hline & & & 80 & 50 & $52073(9.29)$ & 50 & $82551(2.72)$ \\
\hline & & \multirow[t]{3}{*}{0.7} & 40 & 50 & $37120(13.21)$ & 50 & $59790(3.39)$ \\
\hline & & & 60 & 50 & $56054(12.42)$ & 50 & $87998(3.25)$ \\
\hline & & & 80 & 50 & $73441(9.84)$ & 50 & $117959(2.37)$ \\
\hline & \multirow[t]{6}{*}{0.5} & \multirow[t]{3}{*}{0.5} & 40 & 50 & $34749(20.43)$ & 50 & $39549(6.00)$ \\
\hline & & & 60 & 50 & $56153(15.41)$ & 50 & $61690(4.18)$ \\
\hline & & & 80 & 50 & $75693(14.20)$ & 50 & $85458(5.54)$ \\
\hline & & \multirow[t]{3}{*}{0.7} & 40 & 43 & $90420(21.41)$ & 47 & $124485(7.65)$ \\
\hline & & & 60 & 48 & $173763(24.48)$ & 50 & $197265(9.09)$ \\
\hline & & & 80 & 50 & $215616(15.13)$ & 50 & $272689(7.09)$ \\
\hline & \multirow[t]{6}{*}{0.9} & \multirow[t]{3}{*}{0.5} & 40 & 12 & $29452(43.52)$ & 31 & $33797(11.15)$ \\
\hline & & & 60 & 19 & $58561(24.16)$ & 44 & $51153(3.35)$ \\
\hline & & & 80 & 25 & $105523(22.30)$ & 49 & $78544(3.70)$ \\
\hline & & \multirow[t]{3}{*}{0.7} & 40 & 0 & - & 47 & $109043(4.18)$ \\
\hline & & & 60 & 0 & - & 46 & $253284(3.30)$ \\
\hline & & & 80 & 0 & - & 48 & $456783(3.21)$ \\
\hline
\end{tabular}

\subsection{The mixing strategy of low and high crossover rates}

From the results of the previous experiment, low and high crossover rates are suitable for different types of functions. In this second preliminary experiment, mixing both low and high crossover rates, $C R$ in $[0,0.1]$ and $[0.9,1.0]$, is aimed to enhance the basic DE to solve various types of functions. Two mixing strategies are investigated: the switching of low and high crossover rates with proportion 50:50, denoted by MX5050, and the adaptive proportion of crossover rates based on success counts in selection for trial vectors generated from associated strategies, denoted by MX-A. Two strategies for scaling factors are also investigated: the fixed value $F=0.5$ and the random values 
Table 4: Performances of four enhanced DE algorithms using the mixing crossover strategies averaged over 50 independent runs.

\begin{tabular}{|c|c|c|c|c|c|c|c|}
\hline \multirow[t]{2}{*}{ Functions } & \multirow[t]{2}{*}{$C R$} & \multirow[t]{2}{*}{$F$} & \multirow[t]{2}{*}{$N P$} & \multicolumn{2}{|r|}{$D=10$} & \multicolumn{2}{|r|}{$D=30$} \\
\hline & & & & $N S$ & Mean $\mathrm{nf}(\% \mathrm{SD})$ & $N S$ & Mean $\mathrm{nf}(\% \mathrm{SD})$ \\
\hline \multirow[t]{12}{*}{ Schwefel 1.2} & MX5050 & 0.5 & 30 & 50 & $35875(59.55)$ & 50 & $389496(14.66)$ \\
\hline & & & 40 & 50 & $23364(9.36)$ & 50 & $296499(10.46)$ \\
\hline & & & 50 & 50 & $28906(3.65)$ & 50 & $281512(6.90)$ \\
\hline & & {$[0.5,0.7]$} & 30 & 50 & $21775(3.98)$ & 50 & $208189(7.02)$ \\
\hline & & & 40 & 50 & $30943(3.49)$ & 50 & $233574(4.54)$ \\
\hline & & & 50 & 50 & $40757(2.80)$ & 50 & $277860(5.00)$ \\
\hline & MX-A & 0.5 & 30 & 23 & $37818(33.41)$ & 17 & $504692(29.25)$ \\
\hline & & & 40 & 50 & $23401(23.27)$ & 50 & $306201(9.95)$ \\
\hline & & & 50 & 50 & $23413(6.33)$ & 50 & $263027(7.38)$ \\
\hline & & {$[0.5,0.7]$} & 30 & 50 & $19723(7.55)$ & 50 & $197665(7.22)$ \\
\hline & & & 40 & 50 & $26043(4.03)$ & 50 & $216883(7.10)$ \\
\hline & & & 50 & 50 & $34309(2.83)$ & 50 & $255311(4.73)$ \\
\hline \multirow[t]{12}{*}{ Rosenbrock } & MX5050 & 0.5 & 30 & 0 & - & 0 & - \\
\hline & & & 40 & 15 & $577374(57.15)$ & 0 & - \\
\hline & & & 50 & 48 & $112695(38.86)$ & 43 & $2270817(25.41)$ \\
\hline & & {$[0.5,0.7]$} & 30 & 48 & 40953(19.49) & 45 & $788691(21.04)$ \\
\hline & & & 40 & 50 & $46745(8.22)$ & 49 & $285563(8.74)$ \\
\hline & & & 50 & 49 & $56012(6.19)$ & 50 & $307777(5.05)$ \\
\hline & MX-A & 0.5 & 30 & 1 & $22513(-)$ & 0 & - \\
\hline & & & 40 & 3 & $353888(155.41)$ & 6 & $1635769(64.71)$ \\
\hline & & & 50 & 50 & $145829(45.60)$ & 50 & $913004(22.81)$ \\
\hline & & {$[0.5,0.7]$} & 30 & 50 & $41709(14.26)$ & 50 & $278506(15.76)$ \\
\hline & & & 40 & 50 & $49077(7.31)$ & 50 & $314795(7.93)$ \\
\hline & & & 50 & 50 & $59840(5.34)$ & 50 & $388859(4.71)$ \\
\hline \multirow[t]{12}{*}{ Rastrigin } & MX5050 & 0.5 & 30 & 50 & $14942(4.62)$ & 50 & $55173(3.73)$ \\
\hline & & & 40 & 50 & $20404(3.06)$ & 50 & $74952(2.02)$ \\
\hline & & & 50 & 50 & $25564(2.92)$ & 50 & $96372(2.29)$ \\
\hline & & {$[0.5,0.7]$} & 30 & 50 & $18169(4.02)$ & 50 & $71442(2.97)$ \\
\hline & & & 40 & 50 & $24711(4.58)$ & 50 & $99587(3.08)$ \\
\hline & & & 50 & 50 & $314745(3.67)$ & 50 & $128158(2.53)$ \\
\hline & MX-A & 0.5 & 30 & 5 & $14065(3.29)$ & 49 & $45807(2.06)$ \\
\hline & & & 40 & 50 & $18460(2.98)$ & 50 & $60473(1.87)$ \\
\hline & & & 50 & 50 & $22633(2.87)$ & 50 & $75045(1.33)$ \\
\hline & & {$[0.5,0.7]$} & 30 & 50 & $16450(3.81)$ & 49 & $54353(2.08)$ \\
\hline & & & 40 & 50 & $21312(3.56)$ & 50 & $71307(2.19)$ \\
\hline & & & 50 & 50 & $26155(2.70)$ & 50 & $88617(1.47)$ \\
\hline \multirow[t]{12}{*}{ Griewank } & MX5050 & 0.5 & 30 & 45 & $22001(18.91)$ & 32 & $28308(7.14)$ \\
\hline & & & 40 & 49 & $30577(14.46)$ & 42 & $39194(4.10)$ \\
\hline & & & 50 & 50 & $38251(12.85)$ & 49 & $52830(4.88)$ \\
\hline & & {$[0.5,0.7]$} & 30 & 42 & $28994(15.65)$ & 46 & $40445(4.94)$ \\
\hline & & & 40 & 50 & $39983(12.81)$ & 47 & $61878(4.17)$ \\
\hline & & & 50 & 48 & $50993(11.42)$ & 50 & $82976(3.53)$ \\
\hline & MX-A & 0.5 & 30 & 49 & $21928(16.42)$ & 50 & $32404(4.33)$ \\
\hline & & & 40 & 50 & $28303(11.10)$ & 50 & $45151(3.21)$ \\
\hline & & & 50 & 50 & $36129(9.08)$ & 50 & $56892(2.55)$ \\
\hline & & {$[0.5,0.7]$} & 30 & 49 & $26372(14.32)$ & 50 & $40576(3.59)$ \\
\hline & & & 40 & 50 & $35463(11.70)$ & 50 & $53840(2.30)$ \\
\hline & & & 50 & 50 & $44551(12.03)$ & 50 & $67674(2.63)$ \\
\hline
\end{tabular}


in the range of $[0.5,0.7]$. Aiming for smaller sizes of population, $N P=30,40,50$ are varied. The test functions and other settings are the same as in the first preliminary experiment.

Table 4 presents the performance comparison of DE with MX0505 and DE with MX-A. The results show that all four enhanced DE algorithms significantly improve the performance of the basic DE for $N P=40$ (as shown in Tables 2 and 3 of the first experiment). All enhanced DE algorithms can solve all functions for almost all cases of $N P$ and $D$ except for a few cases of $N P=30$. For convenient comparison among these variants, we summarize the results of Table 4 into Table 5 based on the number of successful runs; the notation " $\mathrm{S}$ " stands for $N S=50$ and " $\mathrm{x}$ " stands for $N S$ less than 50. From Table 5, only MX-A with $F=0.5$ and $N P=50$, MX-A with $F \in[0.5,0.7]$ and $N P=40$, and MX-A with $F \in[0.5,0.7]$ and $N P=50$ can solve all test functions. Considering the least number of function evaluations, it is clear that DE with MX-A using $F \in[0.5,0.7]$ and $N P=40$ gives the best performance. This setting is chosen for the proposed DEASC algorithm.

Table 5: Summary results of the successful runs of Table 4.

\begin{tabular}{|c|c|c|c|c|c|c|c|c|c|c|}
\hline \multirow[t]{2}{*}{$C R$} & \multirow[t]{2}{*}{$F$} & \multirow[t]{2}{*}{$N P$} & \multicolumn{2}{|c|}{ Schwefel 1.2} & \multicolumn{2}{|c|}{ Rosenbrock } & \multicolumn{2}{|c|}{ Rastrigin } & \multicolumn{2}{|c|}{ Griewank } \\
\hline & & & $D=10$ & $D=30$ & $D=10$ & $D=30$ & $D=10$ & $D=30$ & $D=10$ & $D=30$ \\
\hline \multirow[t]{6}{*}{ MX0505 } & 0.5 & 30 & $\mathrm{~S}$ & $\mathrm{~S}$ & $\mathrm{x}$ & $\mathrm{x}$ & $\mathrm{S}$ & $\mathrm{S}$ & $\mathrm{x}$ & $\mathrm{x}$ \\
\hline & & 40 & $\mathrm{~S}$ & $\mathrm{~S}$ & $\mathrm{x}$ & $\mathrm{x}$ & $\mathrm{S}$ & $\mathrm{S}$ & $\mathrm{x}$ & $\mathrm{x}$ \\
\hline & & 50 & $\mathrm{~S}$ & $\mathrm{~S}$ & $\mathrm{x}$ & $\mathrm{x}$ & $\mathrm{S}$ & $\mathrm{S}$ & $\mathrm{S}$ & $\mathrm{x}$ \\
\hline & $0.5,0.7$ & 30 & $\mathrm{~S}$ & $\mathrm{~S}$ & $\mathrm{x}$ & $\mathrm{x}$ & $\mathrm{S}$ & $\mathrm{S}$ & $\mathrm{x}$ & $\mathrm{x}$ \\
\hline & & 40 & $\mathrm{~S}$ & $\mathrm{~S}$ & $\mathrm{~S}$ & $\mathrm{x}$ & $\mathrm{S}$ & $\mathrm{S}$ & $\mathrm{S}$ & $\mathrm{x}$ \\
\hline & & 50 & $\mathrm{~S}$ & $\mathrm{~S}$ & $\mathrm{x}$ & $\mathrm{S}$ & $\mathrm{S}$ & $\mathrm{S}$ & $\mathrm{x}$ & $\mathrm{S}$ \\
\hline \multirow[t]{12}{*}{ MX-A } & 0.5 & 30 & $\mathrm{x}$ & $\mathrm{x}$ & $\mathrm{x}$ & $\mathrm{x}$ & $\mathrm{x}$ & $\mathrm{x}$ & $\mathrm{x}$ & $\mathrm{S}$ \\
\hline & & 40 & $\mathrm{~S}$ & $\mathrm{~S}$ & $\mathrm{x}$ & $\mathrm{x}$ & $\mathrm{S}$ & $\mathrm{S}$ & $\mathrm{S}$ & $\mathrm{S}$ \\
\hline & & 50 & $\mathrm{~S}$ & $\mathrm{~S}$ & $\mathrm{~S}$ & $\mathrm{~S}$ & $\mathrm{~S}$ & $\mathrm{~S}$ & $\mathrm{~S}$ & $\mathrm{~S}$ \\
\hline & & & 23413 & 263027 & 145829 & 913004 & 22633 & 75045 & 36126 & 56892 \\
\hline & & & (6.33) & $(7.38)$ & $(45.60)$ & $(22.81)$ & $(2.87)$ & $(1.33)$ & $(9.08)$ & $(2.55)$ \\
\hline & {$[\overline{0.5,0.7}]$} & 30 & $\mathrm{~S}$ & $\mathrm{~S}$ & $\mathrm{~S}$ & $\mathrm{~S}$ & $\mathrm{~S}$ & $\mathrm{x}$ & $\mathrm{x}$ & $\mathrm{S}$ \\
\hline & & 40 & $\mathrm{~S}$ & $\mathrm{~S}$ & $\mathrm{~S}$ & $\mathrm{~S}$ & $\mathrm{~S}$ & $\mathrm{~S}$ & $\mathrm{~S}$ & $\mathrm{~S}$ \\
\hline & & & 26043 & 216883 & 49077 & 314795 & 21312 & 71307 & 35463 & 53840 \\
\hline & & & $(4.03)$ & $(7.10)$ & $(7.31)$ & $(7.93)$ & (3.56) & (2.19) & (11.70) & $(2.30)$ \\
\hline & & 50 & $\mathrm{~S}$ & $\mathrm{~S}$ & $\mathrm{~S}$ & $\mathrm{~S}$ & $\mathrm{~S}$ & $\mathrm{~S}$ & $\mathrm{~S}$ & $\mathrm{~S}$ \\
\hline & & & 34309 & 255311 & 59840 & 388859 & 26155 & 88617 & 44551 & 67674 \\
\hline & & & $(2.83)$ & $(4.73)$ & $(5.34)$ & $(4.71)$ & $(2.70)$ & (1.47) & (12.03) & (2.63) \\
\hline
\end{tabular}

\section{Comparison experiments and results}

\subsection{Performance comparison of DEASC and basic DE}

From the results of the two preliminary experiments, we obtain the proposed differential evolution algorithm with adaptation of switching crossover strategies (DEASC) using the control parameters $N P=40, F$ in [0.5,0.7] and the adaptive crossover rates $C R$ in $[0,0.1]$ and $[0.9,1]$ as described in section 3 . In this experiment, the performances of DEASC are compared with those of two basic DE algorithms: DE50 using $N P=50$ and DE100 using $N P=100$. The recommended control parameter 
$F=0.5$ and $C R=0.9$ are set for both DE50 and DE100 [24]. The dimensions are varied as $D=5,10,30,50,100$ for all test functions.

The performance comparison is presented in Table 6 . The best values are highlighted in boldface. The results show that the DEASC gives the most successful runs for all test functions i.e., $N S=50$ for 37 out of all 40 cases and $N S=48,49,49$ for the remaining 3 cases. It provides the best results for Rosenbrock, Rastrigin, Schwefel, and Griewank functions at all dimensions and the best results for the remaining functions at $D=100$. The basic DE50 gives the best results for Sphere, Schwefel 1.2, Schwefel 2.22, and Ackley functions at $D=5,10,30$ but it performs poorly at high dimensions $(D=50,100)$ for almost all test functions. In addition, DE50 gives $N S=0$ for Rosenbrock, Rastrigin, Schwefel, and Ackley functions at $D=100$. Aiming to solve the convergence problems of DE50, the DE100 with double size of population is tested and compared. DE100 gives better convergence performance than that of DE50 on Rosenbrock, Ackley, and Griewank functions but still cannot improve the convergence performance on Rastrigin and Schwefel functions. In addition, DE100 does not give the best result for any cases.

The Welch t-test at a 0.05 level of significance is also used to compare the performances of DEASC with those of DE50 and DE100 in this order and the results are presented in Table 7 . The values,+ 0 , and - denote that DEASC performs significantly better than, similarly to, and worse than a compared method. The value $\oplus$ denotes that our DEASCs solutions can reach $V T R=10^{-10}$ while those of the compared methods cannot. We conclude from these two tables that the proposed algorithm greatly enhances the basic DE. The performance of DEASC at high dimensions is also remarkable.

\subsection{Performance comparison of DEASC and some adaptive DE variants}

To further verify the performances of the proposed algorithm, the comparison of DEASC and some adaptive DE variants is conducted in this experiment. DEASC is compared with the well-known SaDE, jDE, and JADE algorithms first for 30dimensional and 100-dimensional functions, and then compared with the recently developed SFDE algorithm for 30-dimensional functions. The maximum number of function evaluations $(\operatorname{maxn} f)$ are set the same as the original corresponding settings in [30] and in [12], respectively. Each algorithm is performed 50 independent runs. The mean of best function values (Mean fb) and the standard deviations (SD) are reported.

The performance comparison of DEASC with SaDE, jDE, and JADE is shown in Table 8. The best results are indicated in bold where the results of the compared methods are taken from [30]. The Welch t-test at a significance level of 0.05 is used to compare the performance of DEASC with SaDE, jDE, and JADE in this order and the results are presented in Table 9 . From both tables, the results show that DEASC outperforms jDE for all cases and outperforms SaDE for almost all cases except for Schwefel 1.2. When compared with JADE on 30-dimensional functions, DEASC gives 
Table 6: Performance comparison of DEASC and DE at $V T R=10^{-10}$ averaged over 50 independent runs.

\begin{tabular}{|c|c|c|c|c|c|c|c|}
\hline \multirow[t]{2}{*}{ Functions } & \multirow[t]{2}{*}{$D$} & \multicolumn{2}{|r|}{ DE50 } & \multicolumn{2}{|r|}{ DE100 } & \multicolumn{2}{|r|}{ DEASC } \\
\hline & & $N S$ & Mean $n f(\%$ SD $)$ & $N S$ & Mean $n f(\%$ SD $)$ & $N S$ & Mean $\mathrm{nf}(\% \mathrm{SD})$ \\
\hline \multirow[t]{5}{*}{ Sphere } & 5 & 50 & $6141(4.25)$ & 50 & 12491(3.39) & 50 & $6867(4.20)$ \\
\hline & 10 & 50 & $13128(3.24)$ & 50 & $29228(2.73)$ & 50 & $15853(2.69)$ \\
\hline & 30 & 50 & $39045(3.04)$ & 50 & $107807(2.12)$ & 50 & $51053(1.08)$ \\
\hline & 50 & 50 & $70848(7.25)$ & 50 & $168551(2.08)$ & 50 & $85549(1.00)$ \\
\hline & 100 & 50 & $194710(7.93)$ & 50 & $311610(2.03)$ & 50 & $176270(0.67)$ \\
\hline \multirow[t]{5}{*}{ Schwefel 1.2} & 5 & 50 & $7486(4.46)$ & 50 & $15412(2.52)$ & 50 & $9576(4.34)$ \\
\hline & 10 & 50 & $21318(5.33)$ & 50 & $49425(2.49)$ & 50 & $26131(3.99)$ \\
\hline & 30 & 50 & $219114(7.00)$ & 50 & $452361(2.82)$ & 50 & $220533(5.76)$ \\
\hline & 50 & 50 & $676965(4.69)$ & 50 & $1602178(3.13)$ & 50 & $622164(4.56)$ \\
\hline & 100 & 50 & $3290852(4.26)$ & 50 & $9810692(2.62)$ & 50 & $2956029(4.34)$ \\
\hline \multirow[t]{5}{*}{ Rosenbrock } & 5 & 39 & $77318(140.02)$ & 50 & $25276(8.89)$ & 50 & $15933(8.68)$ \\
\hline & 10 & 49 & $193349(53.72)$ & 49 & $75798(4.60)$ & 49 & $47789(8.33)$ \\
\hline & 30 & 7 & $2475978(13.19)$ & 50 & $410041(3.57)$ & 50 & $321370(8.06)$ \\
\hline & 50 & 0 & - & 46 & $1016873(5.77)$ & 50 & $704574(6.36)$ \\
\hline & 100 & 0 & - & 35 & $5127330(17.2)$ & 50 & $2115876(5.16)$ \\
\hline \multirow[t]{5}{*}{ Schwefel 2.22} & 5 & 50 & $10173(2.81)$ & 50 & $20888(2.27)$ & 50 & $10674(2.67)$ \\
\hline & 10 & 50 & $21978(2.79)$ & 50 & $49329(1.72)$ & 50 & $23897(1.31)$ \\
\hline & 30 & 50 & $62004(2.31)$ & 50 & $186542(1.78)$ & 50 & $74763(0.93)$ \\
\hline & 50 & 50 & $98283(3.25)$ & 50 & $287723(1.89)$ & 50 & $124250(0.74)$ \\
\hline & 100 & 50 & $265381(11.32)$ & 50 & $476415(1.60)$ & 50 & $251762(0.53)$ \\
\hline \multirow[t]{5}{*}{ Rastrigin } & 5 & 49 & $21392(13.76)$ & 50 & $49882(9.31)$ & 50 & $9689(4.62)$ \\
\hline & 10 & 24 & $78415(26.15)$ & 46 & $318351(29.65)$ & 50 & $21299(3.33)$ \\
\hline & 30 & 0 & - & 0 & - & 50 & $71135(2.03)$ \\
\hline & 50 & 0 & - & 0 & - & 50 & $133372(1.76)$ \\
\hline & 100 & 0 & - & 0 & - & 50 & $357429(1.42)$ \\
\hline \multirow[t]{5}{*}{ Schwefel } & 5 & 50 & $11148(10.75)$ & 50 & $23383(6.78)$ & 50 & $8583(3.73)$ \\
\hline & 10 & 50 & $41147(17.14)$ & 50 & $102899(12.2)$ & 50 & $19444(2.68)$ \\
\hline & 30 & 1 & 206999(-) & 16 & $853080(13.99)$ & 50 & $61469(1.42)$ \\
\hline & 50 & 0 & - & 0 & - & 49 & $103618(1.32)$ \\
\hline & 100 & 0 & - & 0 & - & 48 & $228704(1.02)$ \\
\hline \multirow[t]{5}{*}{ Ackley } & 5 & 50 & $10550(3.39)$ & 50 & $21796(1.86)$ & 50 & $11932(2.27)$ \\
\hline & 10 & 50 & $21923(2.49)$ & 50 & $49049(1.79)$ & 50 & $26274(1.85)$ \\
\hline & 30 & 50 & $62965(2.89)$ & 50 & $173346(1.80)$ & 50 & $81516(0.98)$ \\
\hline & 50 & 32 & $112678(4.12)$ & 50 & $269773(1.68)$ & 50 & $134637(0.84)$ \\
\hline & 100 & 0 & - & 50 & $488027(2.04)$ & 50 & $272335(0.61)$ \\
\hline \multirow[t]{5}{*}{ Griewank } & 5 & 37 & $39811(14.35)$ & 50 & $98935(9.14)$ & 50 & $23789(11.38)$ \\
\hline & 10 & 12 & $37808(22.51)$ & 37 & $186967(21.11)$ & 50 & $36531(10.6)$ \\
\hline & 30 & 40 & $40209(3.47)$ & 50 & $110873(2.12)$ & 50 & $54102(3.14)$ \\
\hline & 50 & 36 & $69589(7.18)$ & 49 & $169617(1.96)$ & 50 & $86964(1.42)$ \\
\hline & 100 & 23 & $186861(6.77)$ & 47 & $306174(2.34)$ & 50 & $174762(0.93)$ \\
\hline
\end{tabular}


Table 7: Statistical significance test for comparing DEASC with DE50 and DE100 at $V T R=10^{-10}$ averaged over 50 independent runs.

\begin{tabular}{lccccc}
\hline Function & $D$ & Significance & Function & $D$ & Significance \\
\hline Sphere & 5 &,-+ & Rastrigin & 5 &,++ \\
& 10 &,-+ & & 10 &,++ \\
& 30 &,-+ & & 30 & $\oplus, \oplus$ \\
& 50 &,-+ & & 50 & $\oplus, \oplus$ \\
& 100 &,++ & & 100 & $\oplus, \oplus$ \\
\hline Schwefel 1.2 & 5 &,-+ & Schwefel & 5 &,++ \\
& 10 &,-+ & & 10 &,++ \\
& 30 & $0,+$ & & 30 &,++ \\
& 50 &,++ & & 50 & $\oplus, \oplus$ \\
& 100 &,++ & & 100 & $\oplus, \oplus$ \\
\hline Rosenbrock & 5 &,++ & Ackley & 5 &,-+ \\
& 10 &,++ & & 10 &,-+ \\
& 30 &,++ & & 30 &,-+ \\
& 50 & $\oplus,+$ & & 50 &,++ \\
& 100 & $\oplus,+$ & & 100 & $\oplus,+$ \\
\hline Schwefel 2.22 & 5 &,-+ & Griewank & 5 &,++ \\
& 10 &,-+ & & 10 &,++ \\
& 30 &,-+ & & 30 &,++ \\
& 50 &,-+ & & 50 &,++ \\
& 100 &,++ & & 100 &,++ \\
\hline
\end{tabular}

the best results for 5 out of all 8 functions while JADE gives the best results for the remaining 3 functions. For high dimension $D=100$, the DEASC performs better than JADE for all cases except for Schwefel 1.2 function. It is clear that DEASC overall outperforms these three compared methods. It is observed again that DEASC shows outstanding performance on high dimensional test functions.

Table 10 presents the performance comparison of DEASC and SFDE. The best values of SFDE are taken from the original paper [12]. The last column of the table shows the performances of DEASC compared with SFDE using the Welch t-test at a 0.05 level of significance. The results show that the proposed DEASC performs better than SFDE for 4 functions especially for Rosenbrock, Rastrigin, and Schwefel functions, and performs similarly for 2 cases, and performs worse than SFDE for 2 cases. This indicates that DEASC algorithm overall outperforms SFDE algorithm. 
Table 8: Performance comparison of DEASC and SaDE, jDE, JADE averaged over 50 independent runs.

\begin{tabular}{|c|c|c|c|c|c|}
\hline Function & $\operatorname{maxnf}$ & $\begin{array}{c}\text { SaDE } \\
\text { Mean fb } \\
(\mathrm{SD})\end{array}$ & $\begin{array}{c}\text { jDE } \\
\text { Mean fb } \\
(\mathrm{SD})\end{array}$ & $\begin{array}{c}\text { JADE } \\
\text { Mean fb } \\
(\mathrm{SD})\end{array}$ & $\begin{array}{c}\text { DEASC } \\
\text { Mean fb } \\
(\mathrm{SD})\end{array}$ \\
\hline \multicolumn{6}{|l|}{$D=30$} \\
\hline Sphere & $1.5 \times 10^{5}$ & $\begin{array}{c}4.50 \mathrm{E}-20 \\
(6.90 \mathrm{E}-20)\end{array}$ & $\begin{array}{c}2.50 \mathrm{E}-28 \\
(3.50 \mathrm{E}-28)\end{array}$ & $\begin{array}{c}1.80 \mathrm{E}-60 \\
(8.40 \mathrm{E}-60)\end{array}$ & $\begin{array}{c}1.75 \mathrm{E}-39 \\
(9.52 \mathrm{E}-40)\end{array}$ \\
\hline Schwefel 1.2 & $5 \times 10^{5}$ & $\begin{array}{c}9.00 \mathrm{E}-37 \\
(5.43 \mathrm{E}-36)\end{array}$ & $\begin{array}{c}5.20 \mathrm{E}-14 \\
(1.10 \mathrm{E}-13)\end{array}$ & $\begin{array}{c}5.70 E-61 \\
(2.70 E-60)\end{array}$ & $\begin{array}{c}1.01 \mathrm{E}-27 \\
(4.51 \mathrm{E}-27)\end{array}$ \\
\hline Rosenbrock & $3 \times 10^{5}$ & $\begin{array}{c}2.10 \mathrm{E}+01 \\
(7.80 \mathrm{E}+00)\end{array}$ & $\begin{array}{c}1.30 \mathrm{E}+01 \\
(1.40 \mathrm{E}+01)\end{array}$ & $\begin{array}{c}8.00 \mathrm{E}-02 \\
(5.60 \mathrm{E}-01)\end{array}$ & $\begin{array}{c}\text { 5.89E-06 } \\
(3.13 \mathrm{E}-05)\end{array}$ \\
\hline Schwefel 2.22 & $2 \times 10^{5}$ & $\begin{array}{c}1.90 \mathrm{E}-14 \\
(1.05 \mathrm{E}-14)\end{array}$ & $\begin{array}{c}1.50 \mathrm{E}-23 \\
(1.00 \mathrm{E}-23)\end{array}$ & $\begin{array}{c}1.80 \mathrm{E}-25 \\
(8.80 \mathrm{E}-25)\end{array}$ & $\begin{array}{c}4.33 \mathrm{E}-31 \\
(1.75 \mathrm{E}-31)\end{array}$ \\
\hline Rastrigin & $1 \times 10^{5}$ & $\begin{array}{c}1.20 \mathrm{E}-03 \\
(6.50 \mathrm{E}-04)\end{array}$ & $\begin{array}{c}1.50 \mathrm{E}-04 \\
(2.00 \mathrm{E}-04)\end{array}$ & $\begin{array}{c}1.00 \mathrm{E}-04 \\
(6.00 \mathrm{E}-05)\end{array}$ & $\begin{array}{c}0 \\
(0)\end{array}$ \\
\hline Schwefel & $1 \times 10^{5}$ & $\begin{array}{c}4.70 \mathrm{E}+00 \\
(3.30 \mathrm{E}+01)\end{array}$ & $\begin{array}{c}7.90 \mathrm{E}-11 \\
(1.30 \mathrm{E}-10)\end{array}$ & $\begin{array}{c}3.30 \mathrm{E}-05 \\
(2.30 \mathrm{E}-05)\end{array}$ & $\begin{array}{c}0 \\
(0)\end{array}$ \\
\hline Ackley & $5 \times 10^{4}$ & $\begin{array}{c}2.70 \mathrm{E}-03 \\
(5.10 \mathrm{E}-04)\end{array}$ & $\begin{array}{c}3.50 \mathrm{E}-04 \\
(1.00 \mathrm{E}-04)\end{array}$ & $\begin{array}{c}8.20 \mathrm{E}-10 \\
(6.90 \mathrm{E}-10)\end{array}$ & $\begin{array}{c}3.85 \mathrm{E}-06 \\
(8.38 \mathrm{E}-07)\end{array}$ \\
\hline Griewank & $5 \times 10^{4}$ & $\begin{array}{c}7.80 \mathrm{E}-04 \\
(1.20 \mathrm{E}-03)\end{array}$ & $\begin{array}{c}1.90 \mathrm{E}-05 \\
(5.80 \mathrm{E}-05)\end{array}$ & $\begin{array}{c}9.90 \mathrm{E}-08 \\
(6.00 \mathrm{E}-07)\end{array}$ & $\begin{array}{c}4.32 \mathrm{E}-09 \\
(9.84 \mathrm{E}-09)\end{array}$ \\
\hline$D=100$ & & & & & \\
\hline Sphere & $8 \times 10^{5}$ & $\begin{array}{c}2.90 \mathrm{E}-08 \\
(3.20 \mathrm{E}-08)\end{array}$ & $\begin{array}{c}5.00 \mathrm{E}-15 \\
(1.70 \mathrm{E}-15)\end{array}$ & $\begin{array}{c}1.20 \mathrm{E}-48 \\
(1.50 \mathrm{E}-48)\end{array}$ & $\begin{array}{c}1.47 \mathrm{E}-64 \\
(1.10 \mathrm{E}-64)\end{array}$ \\
\hline Schwefel 1.2 & $3.2 \times 10^{6}$ & $\begin{array}{c}2.40 \mathrm{E}-13 \\
(5.20 \mathrm{E}-13)\end{array}$ & $\begin{array}{c}5.40 \mathrm{E}-02 \\
(2.70 \mathrm{E}-02)\end{array}$ & $\begin{array}{c}1.20 \mathrm{E}-26 \\
(2.00 \mathrm{E}-26)\end{array}$ & $\begin{array}{c}5.58 \mathrm{E}-11 \\
(1.58 \mathrm{E}-11)\end{array}$ \\
\hline Rosenbrock & $2.4 \times 10^{6}$ & $\begin{array}{c}9.40 \mathrm{E}+01 \\
(4.00 \mathrm{E}-01)\end{array}$ & $\begin{array}{c}7.20 \mathrm{E}+01 \\
(1.10 \mathrm{E}+01)\end{array}$ & $\begin{array}{c}5.60 \mathrm{E}-01 \\
(1.40 \mathrm{E}+00)\end{array}$ & $\begin{array}{c}2.00 \mathrm{E}-15 \\
(8.41 \mathrm{E}-15)\end{array}$ \\
\hline Schwefel 2.22 & $1.2 \times 10^{6}$ & $\begin{array}{c}1.70 \mathrm{E}-05 \\
(3.80 \mathrm{E}-06)\end{array}$ & $\begin{array}{c}4.10 \mathrm{E}-15 \\
(1.10 \mathrm{E}-15)\end{array}$ & $\begin{array}{c}1.10 \mathrm{E}-41 \\
(5.10 \mathrm{E}-41)\end{array}$ & $\begin{array}{c}2.38 E-58 \\
(8.28 E-59)\end{array}$ \\
\hline Rastrigin & $1.2 \times 10^{6}$ & $\begin{array}{c}9.10 \mathrm{E}-03 \\
(1.80 \mathrm{E}-03)\end{array}$ & $\begin{array}{c}2.10 \mathrm{E}-04 \\
(2.10 \mathrm{E}-04)\end{array}$ & $\begin{array}{c}1.90 \mathrm{E}-01 \\
(3.80 \mathrm{E}-02)\end{array}$ & $\begin{array}{c}2.65 \mathrm{E}-14 \\
(4.89 \mathrm{E}-14)\end{array}$ \\
\hline Schwefel & $4 \times 10^{5}$ & $\begin{array}{c}5.40 \mathrm{E}+03 \\
(3.70 \mathrm{E}+02)\end{array}$ & $\begin{array}{c}4.90 \mathrm{E}+03 \\
(4.10 \mathrm{E}+02)\end{array}$ & $\begin{array}{c}8.90 \mathrm{E}+03 \\
(3.00 \mathrm{E}+02)\end{array}$ & $\begin{array}{c}1.01 \mathrm{E}-10 \\
(2.22 \mathrm{E}-12)\end{array}$ \\
\hline Ackley & $2 \times 10^{5}$ & $\begin{array}{c}1.60 \mathrm{E}+00 \\
(1.20 \mathrm{E}-01)\end{array}$ & $\begin{array}{c}8.50 \mathrm{E}-01 \\
(1.20 \mathrm{E}-01)\end{array}$ & $\begin{array}{c}7.90 \mathrm{E}-06 \\
(2.60 \mathrm{E}-06)\end{array}$ & $\begin{array}{c}1.37 \mathrm{E}-07 \\
(1.80 \mathrm{E}-08)\end{array}$ \\
\hline Griewank & $2 \times 10^{5}$ & $\begin{array}{c}1.10 \mathrm{E}+00 \\
(1.80 \mathrm{E}-02)\end{array}$ & $\begin{array}{c}1.10 \mathrm{E}+00 \\
(2.00 \mathrm{E}-02)\end{array}$ & $\begin{array}{c}3.90 \mathrm{E}-04 \\
(2.00 \mathrm{E}-03)\end{array}$ & $\begin{array}{c}6.71 \mathrm{E}-13 \\
(1.55 \mathrm{E}-13)\end{array}$ \\
\hline
\end{tabular}

Table 9: Statistical significance test for comparing DEASC with SaDE, jDE and JADE averaged over 50 independent runs.

\begin{tabular}{lrclrc}
\hline Function & maxnf & Significance & Function & $\operatorname{maxnf}$ & Significance \\
\hline$D=30$ & & & $D=100$ & & \\
Sphere & $1.5 \times 10^{5}$ &,,++- & Sphere & $8 \times 10^{5}$ &,,+++ \\
Schwefel 1.2 & $5 \times 10^{5}$ &,,-+- & Schwefel 1.2 & $3.2 \times 10^{6}$ &,,-+- \\
Rosenbrock & $3 \times 10^{5}$ &,,+++ & Rosenbrock & $2.4 \times 10^{6}$ &,,+++ \\
Schwefel 2.22 & $2 \times 10^{5}$ &,,+++ & Schwefel 2.22 & $1.2 \times 10^{6}$ &,,+++ \\
Rastrigin & $1 \times 10^{5}$ &,,+++ & Rastrigin & $1.2 \times 10^{6}$ &,,+++ \\
Schwefel & $1 \times 10^{5}$ &,,+++ & Schwefel & $4 \times 10^{5}$ &,,+++ \\
Ackley & $5 \times 10^{4}$ &,,++- & Ackley & $2 \times 10^{5}$ &,,+++ \\
Griewank & $5 \times 10^{4}$ &,,+++ & Griewank & $2 \times 10^{5}$ &,,+++ \\
\hline
\end{tabular}


Table 10: Performance comparison of DEASC and SFDE averaged over 50 independent runs for 30-dimensional functions.

\begin{tabular}{|c|c|c|c|c|}
\hline Function & $\operatorname{maxnf}$ & $\begin{array}{c}\text { SFDE } \\
\text { Mean fb } \\
\text { (SD) }\end{array}$ & $\begin{array}{c}\text { DEASC } \\
\text { Mean fb } \\
\text { (SD) }\end{array}$ & Significance \\
\hline Sphere & $1.5 \times 10^{5}$ & $\begin{array}{c}1.91 \mathrm{E}-73 \\
(8.38 \mathrm{E}-73)\end{array}$ & $\begin{array}{c}1.75 \mathrm{E}-39 \\
(9.52 \mathrm{E}-40)\end{array}$ & - \\
\hline Schwefel 1.2 & $5 \times 10^{5}$ & $\begin{array}{l}1.90 \mathrm{E}-11 \\
(6.15 \mathrm{E}-11)\end{array}$ & $\begin{array}{c}1.01 \mathrm{E}-27 \\
(4.51 \mathrm{E}-27)\end{array}$ & + \\
\hline Rosenbrock & $2 \times 10^{6}$ & $\begin{array}{c}2.71 \mathrm{E}-03 \\
(1.65 \mathrm{E}-02)\end{array}$ & $\begin{array}{c}0 \\
(0)\end{array}$ & + \\
\hline Schwefel 2.22 & $2 \times 10^{5}$ & $\begin{array}{c}1.23 \mathrm{E}-53 \\
(3.30 \mathrm{E}-53)\end{array}$ & $\begin{array}{c}4.33 \mathrm{E}-31 \\
(1.75 \mathrm{E}-31)\end{array}$ & - \\
\hline Rastrigin & $5 \times 10^{5}$ & $\begin{array}{c}2.24 \mathrm{E}+00 \\
(1.66 \mathrm{E}+00)\end{array}$ & $\begin{array}{c}0 \\
(0)\end{array}$ & + \\
\hline Schwefel & $9 \times 10^{5}$ & $\begin{array}{c}9.96 \mathrm{E}+01 \\
(1.19 \mathrm{E}+02)\end{array}$ & $\begin{array}{c}0 \\
(0)\end{array}$ & + \\
\hline Ackley & $2 \times 10^{5}$ & $\begin{array}{c}5.77 \mathrm{E}-15 \\
(1.78 \mathrm{E}-15)\end{array}$ & $\begin{array}{c}5.70 \mathrm{E}-15 \\
(1.79 \mathrm{E}-15)\end{array}$ & 0 \\
\hline Griewank & $3 \times 10^{5}$ & $\begin{array}{c}0 \\
(0)\end{array}$ & $\begin{array}{c}0 \\
(0)\end{array}$ & 0 \\
\hline
\end{tabular}

\section{Performance of DEASC on solving systems of nonlinear equations}

Solving systems of nonlinear equations is an important application of continuous optimization $[6,20]$. In this experiment, the DEASC is applied to solve the systems of nonlinear equations described in the following form:

$$
\left\{\begin{aligned}
f_{1}\left(x_{1}, x_{2}, \ldots, x_{n}\right) & =0 \\
f_{2}\left(x_{1}, x_{2}, \ldots, x_{n}\right) & =0 \\
& \vdots \\
f_{m}\left(x_{1}, x_{2}, \ldots, x_{n}\right) & =0
\end{aligned}\right.
$$

where $f_{i}:[L B, U B]^{n} \subseteq R^{n} \rightarrow R$ for $i=1, \ldots, m$ are nonlinear equations (including linear functions) and $x=\left(x_{1}, x_{2}, \ldots, x_{n}\right)$ is a real vector. The aim is to find a solution $x^{*}$ such that $f_{i}\left(x^{*}\right)=0$ simultaneously for all $i$. The problem is transformed into the corresponding optimization problem by defining the objective function $f$ as the sum of the squares of all $f_{i}$ as follows:

$$
f(x)=\sum_{i=1}^{m} f_{i}^{2}(x) .
$$

Four selected practical problems are listed as follows.

Problem 1: Robot kinematics application [16]. This problem concerns the indirectposition problem which is to find the desired position and orientation of the robot hand 
and the relative joint displacements. The problem can be reduced to the following system of eight equations in eight variables

$$
\left\{\begin{aligned}
f_{1}(\mathbf{x})= & 4.731 \times 10^{-3} x_{1} x_{3}-0.3578 x_{2} x_{3}-0.1238 x_{1}+x_{7}-1.637 \times 10^{-3} x_{2} \\
& -0.9338 x_{4}-0.3571=0 \\
f_{2}(\mathbf{x})= & 0.2238 x_{1} x_{3}+0.7623 x_{2} x_{3}+0.2638 x_{1}-0.07745 x_{2}-0.6734 x_{4}-0.6022=0 \\
f_{3}(\mathbf{x})= & x_{6} x_{8}+0.3578 x_{1}+4.731 \times 10^{-3} x_{2}=0 \\
f_{4}(\mathbf{x})= & -0.7623 x_{1}+0.2238 x_{2}+0.3461=0 \\
f_{5}(\mathbf{x})= & x_{1}^{2}+x_{2}^{2}-1=0 \\
f_{6}(\mathbf{x})= & x_{3}^{2}+x_{4}^{2}-1=0 \\
f_{7}(\mathbf{x})= & x_{5}^{2}+x_{6}^{2}-1=0 \\
f_{8}(\mathbf{x})= & x_{7}^{2}+x_{8}^{2}-1=0
\end{aligned}\right.
$$

where $-1 \leq x_{i} \leq 1$.

Problem 2: Automotive steering application [9,21]. This problem describes the kinematic synthesis of a trailing six-member mechanism for automotive steering. The system consists of three equations in three unknown as follows

$$
\left\{\begin{aligned}
f_{i}(\mathbf{x})= & {\left[E_{i}\left(x_{2} \sin \phi_{i}-x_{3}\right)-F_{i}\left(x_{2} \sin \psi_{i}-x_{3}\right)\right]^{2} } \\
& +\left[F_{i}\left(1+x_{2} \cos \psi_{i}\right)-E_{i}\left(x_{2} \cos \phi_{i}-1\right)\right]^{2} \\
& -\left[\left(1+x_{2} \cos \psi_{i}\right)\left(x_{2} \sin \phi_{i}-x_{3}\right) x_{1}\right. \\
& \left.-\left(x_{2} \sin \psi_{i}-x_{3}\right)\left(x_{2} \cos \phi_{i}-x_{3}\right) x_{1}\right]^{2}=0, i=1,2,3
\end{aligned}\right.
$$

where $0 \leq x_{i} \leq 1$ and

$$
\begin{aligned}
& E_{i}=x_{2}\left(\cos \psi_{i}-\cos \psi_{0}\right)-x_{2} x_{3}\left(\sin \psi_{i}-\sin \psi_{0}-\left(x_{2} \sin \psi_{i}-x_{3}\right) x_{1}\right. \\
& F_{i}=-x_{2} \cos \phi_{i}-x_{2} x_{3} \sin \phi_{i}+x_{2} \cos \phi_{0}+x_{1} x_{3}+\left(x_{3}-x_{1}\right) x_{2} \sin \phi_{0} .
\end{aligned}
$$

The constants $\phi_{i}$ and $\psi_{i}$ are given as follows

$$
\begin{aligned}
& \phi_{0}=1.3954170041747090114, \phi_{1}=1.7444828545735749268, \\
& \phi_{2}=2.0656234369405315689, \phi_{3}=2.4600678478912500533, \\
& \psi_{0}=1.7461756494150842271, \psi_{1}=2.0364691127919609051, \\
& \psi_{2}=2.2390977868265978920, \psi_{3}=2.4600678409809344550 .
\end{aligned}
$$

Problem 3 : Chemical equilibrium application $[14,16,19]$. This problem describes a chemical equilibrium system. It concerns the combustion of propane in air to form ten products, which are transformed to ten equations in ten variables. The system 
can be reduced to the following systems of five equations in five variables

$$
\left\{\begin{aligned}
f_{1}(\mathbf{x})= & x_{1} x_{2}+x_{1}-3 x_{5}=0 \\
f_{2}(\mathbf{x})= & 2 x_{1} x_{2}+x_{1}+x_{2} x_{3}^{2}+R_{5} x_{2}-R_{1} x_{5}+2 R_{7} x_{2}^{2}+R_{4} x_{2} x_{3}+R_{6} x_{2} x_{4}=0 \\
f_{3}(\mathbf{x})= & 2 x_{2} x_{3}^{2}+2 R_{2} x_{3}^{2}-8 x_{5}+R_{3} x_{3}+R_{4} x_{2} x_{3}=0 \\
f_{4}(\mathbf{x})= & R_{6} x_{2} x_{4}+2 x_{4}^{2}-4 R_{1} x_{5}=0 \\
f_{5}(\mathbf{x})= & x_{1}\left(x_{2}+1\right)+R_{7} x_{2}^{2}+x_{2} x_{3}^{2}+R_{5} x_{2}+R_{2} x_{3}^{2}+x_{4}^{2}-1+R_{3} x_{3} \\
& +R_{4} x_{2} x_{3}+R_{6} x_{2} x_{4}=0
\end{aligned}\right.
$$

where $-100 \leq x_{i} \leq 100$ and the constants used in this system are

$$
\begin{aligned}
& R_{1}=10, R_{2}=0.193, R_{3}=0.002597 / \sqrt{40} \\
& R_{4}=0.003448 / \sqrt{40}, R_{5}=0.00001799 / 40, \\
& R_{6}=0.0002155 / \sqrt{40}, R_{7}=0.00003846 / 40 .
\end{aligned}
$$

Problem 4 : Economics modeling application [19,28]. This problem arises in economics modeling. It can be extended for general dimensions $n$ as follows

$$
\left\{\begin{array}{l}
f_{i}(\mathbf{x})=\left(x_{i}+\sum_{j=1}^{n-i-1} x_{j} x_{j+i}\right) x_{n}-c_{i}=0, i=1,2, \ldots, n-1 \\
f_{n}(\mathbf{x})=\sum_{j=1}^{n-1} x_{j}+1=0
\end{array}\right.
$$

where $c_{i}=0$ for all $i$ as in [19] and $-10 \leq x_{i} \leq 10$.

We set $\operatorname{VTR}=10^{-20}$ and $10^{-30}$ in order to verify that the high quality solution can be obtained. For each problem, the algorithm is performed 50 independent runs. The NS, Mean nf, and \%SD values are reported. The performance of DEASC is presented in Table 11. The results show that DEASC can solve all problems at both $V T R=10^{-20}$ and $V T R=10^{-30}$ with good convergence performances. The convergent graphs are illustrated in Figure 2. Some obtained solutions are also given in Table 12. 
Table 11: Performance of DEASC on solving systems of nonlinear equations averaged over 50 independent runs.

\begin{tabular}{lcccc}
\hline Problems & VTR & NS & Mean nf & \%SD \\
\hline Robot kinematics & $10^{-20}$ & 50 & 39117 & 22.79 \\
application & $10^{-30}$ & 50 & 51806 & 17.08 \\
\hline Automotive steering & $10^{-20}$ & 50 & 4125 & 11.13 \\
application & $10^{-30}$ & 50 & 6427 & 8.08 \\
\hline Chemical equilibrium & $10^{-20}$ & 50 & 62931 & 7.94 \\
application & $10^{-30}$ & 50 & 68962 & 7.23 \\
\hline Economics modeling & $10^{-20}$ & 50 & 13281 & 6.53 \\
application & $10^{-30}$ & 50 & 18139 & 0.13 \\
\hline
\end{tabular}

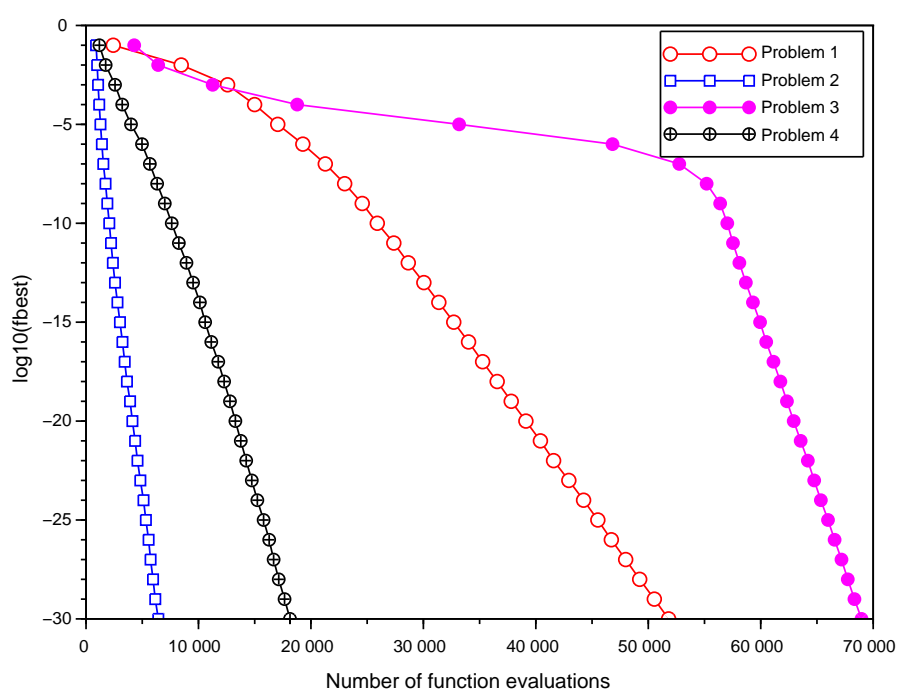

Figure 2: The convergent graphs of DEASC for solving the nonlinear systems.

\section{Discussion}

In section 4.2, the effect of using low and high crossover rate values $C R=0.1$ and 0.9 , and the scaling factor values $F=0.5$ and 0.7 on the performance of basic DE is verified. It has been shown that in this scope of control parameters, the crossover 
Table 12: Some solutions of the nonlinear systems.

\begin{tabular}{|c|c|c|c|c|c|}
\hline Problems & & Solution 1 & Solution 2 & Solution 3 & Solution 4 \\
\hline Robot & $x_{1}$ & $6.715543 \mathrm{E}-01$ & $6.715543 \mathrm{E}-01$ & $1.644317 \mathrm{E}-01$ & $1.644317 \mathrm{E}-01$ \\
\hline kinematics & $x_{2}$ & 7.409554E-01 & 7.409554E-01 & $-9.863885 \mathrm{E}-01$ & $-9.863885 \mathrm{E}-01$ \\
\hline \multirow[t]{6}{*}{ application } & $x_{3}$ & $-2.396117 \mathrm{E}-01$ & $-2.396117 \mathrm{E}-01$ & 2.396160E-01 & $2.396160 \mathrm{E}-01$ \\
\hline & $x_{4}$ & $-9.708688 \mathrm{E}-01$ & $-9.708688 \mathrm{E}-01$ & $-9.708677 \mathrm{E}-01$ & $-9.708677 \mathrm{E}-01$ \\
\hline & $x_{5}$ & $9.579171 \mathrm{E}-01$ & $-9.579171 \mathrm{E}-01$ & 9.976354E-01 & $9.976354 \mathrm{E}-01$ \\
\hline & $x_{6}$ & $2.870450 \mathrm{E}-01$ & $2.870450 \mathrm{E}-01$ & $-6.872850 \mathrm{E}-02$ & $6.872850 \mathrm{E}-02$ \\
\hline & $x_{7}$ & $-5.279090 \mathrm{E}-01$ & $-5.279090 \mathrm{E}-01$ & $-6.155084 \mathrm{E}-01$ & $-6.155084 \mathrm{E}-01$ \\
\hline & $x_{8}$ & $-8.493009 \mathrm{E}-01$ & $-8.493009 \mathrm{E}-01$ & 7.881303E-01 & $-7.881303 \mathrm{E}-01$ \\
\hline \multirow{3}{*}{$\begin{array}{l}\text { Automotive } \\
\text { steering } \\
\text { application }\end{array}$} & $x_{1}$ & $1.496998 \mathrm{E}-03$ & $-7.500810 \mathrm{E}-04$ & $-3.004333 \mathrm{E}-05$ & $6.341136 \mathrm{E}-04$ \\
\hline & $x_{2}$ & $-2.636339 \mathrm{E}-08$ & $-9.612057 \mathrm{E}-09$ & 9.609970E-08 & 9.921813E-08 \\
\hline & $x_{3}$ & $6.504039 \mathrm{E}-06$ & $1.095419 \mathrm{E}-05$ & $5.928428 \mathrm{E}-04$ & $-2.716409 \mathrm{E}-05$ \\
\hline \multirow{5}{*}{$\begin{array}{l}\text { Chemical } \\
\text { equilibrium } \\
\text { application }\end{array}$} & $x_{1}$ & $3.114102 \mathrm{E}-03$ & $2.757177 \mathrm{E}-03$ & $2.471000 \mathrm{E}-03$ & $2.153308 \mathrm{E}-03$ \\
\hline & $x_{2}$ & $3.459792 \mathrm{E}+01$ & $3.924229 \mathrm{E}+01$ & $4.387922 \mathrm{E}+01$ & $5.054957 \mathrm{E}+01$ \\
\hline & $x_{3}$ & $6.504178 \mathrm{E}-02$ & $-6.138760 \mathrm{E}-02$ & $5.778446 \mathrm{E}-02$ & $-5.414481 \mathrm{E}-02$ \\
\hline & $x_{4}$ & 8.593781E-01 & $8.597244 \mathrm{E}-01$ & $-8.602055 \mathrm{E}-01$ & $-8.606713 \mathrm{E}-01$ \\
\hline & $x_{5}$ & 3.695186E-02 & 3.698504E-02 & 3.696552E-02 & $3.700070 \mathrm{E}-02$ \\
\hline \multirow{10}{*}{$\begin{array}{l}\text { Economics } \\
\text { modeling } \\
\text { application }\end{array}$} & $x_{1}$ & $3.776093 \mathrm{E}+00$ & $-5.930003 \mathrm{E}+00$ & $-1.871494 \mathrm{E}-01$ & $3.140586 \mathrm{E}+00$ \\
\hline & $x_{2}$ & $-4.086607 \mathrm{E}+00$ & $-3.021762 \mathrm{E}+00$ & $-5.435169 \mathrm{E}+00$ & 8.365479E-01 \\
\hline & $x_{3}$ & $-2.431646 \mathrm{E}+00$ & $-1.534828 \mathrm{E}+00$ & $-1.031702 \mathrm{E}+00$ & $-3.555928 \mathrm{E}+00$ \\
\hline & $x_{4}$ & $3.452982 \mathrm{E}-01$ & $3.349701 \mathrm{E}+00$ & $-3.082598 \mathrm{E}+00$ & $-4.180001 \mathrm{E}-01$ \\
\hline & $x_{5}$ & $-4.644880 \mathrm{E}+00$ & $8.061775 \mathrm{E}+00$ & $-5.055115 \mathrm{E}-01$ & $1.824013 \mathrm{E}-01$ \\
\hline & $x_{6}$ & $-4.620380 \mathrm{E}-02$ & $3.792569 \mathrm{E}+00$ & $7.916335 \mathrm{E}+00$ & $-2.000120 \mathrm{E}+00$ \\
\hline & $x_{7}$ & $5.196580 \mathrm{E}+00$ & $-4.868964 \mathrm{E}+00$ & $-4.476051 \mathrm{E}+00$ & $-6.353615 \mathrm{E}+00$ \\
\hline & $x_{8}$ & $2.014114 \mathrm{E}+00$ & $-5.325023 \mathrm{E}+00$ & $5.265743 \mathrm{E}+00$ & $3.486881 \mathrm{E}+00$ \\
\hline & $x_{9}$ & $-1.122749 \mathrm{E}+00$ & $4.476536 \mathrm{E}+00$ & 5.361015E-01 & $3.681248 \mathrm{E}+00$ \\
\hline & $x_{10}$ & 0 & 0 & 0 & 0 \\
\hline
\end{tabular}

rates play an important role in achieving good convergence results and either low or high crossover rate appears to be a suitable choice for different type of functions: low crossover rate $(0.1)$ are suitable for separable and multimodal functions, and high crossover rate (0.9) are suitable for unimodal and nonseparable functions. It is also observed that $F=0.5$ speeds up the convergence but may cause the premature convergence or stagnation especially when $N P$ is low and $D$ is high while $F=0.7$ can prevent such problems for a wide range of $N P$ but requires more computations. From this result, the $F$ values in the range of $[0.5,0.7]$ are utilized in the proposed DEASC algorithm. Note that using $F$ values in this manner was also mentioned in [24]. It provides more diversity during the search process with slightly increasing cost on function evaluations (in the case that $F=0.5$ has already provided a good result).

Two mixing strategies of using low and high crossover rates $C R$ in [0,0.1] and $[0.9,1.0]$ are investigated in section 4.3. As also stated in [15] and confirmed in section 4.2 , the low $C R$ values are useful for highly multimodal functions while the high values are useful for highly nonseparable functions. For unmatched types of functions, using low $C R$ values could slow down the search while using high $C R$ values could lead to stagnation or premature convergence easily. To design an efficient general-purpose optimization algorithm that can solve various types of functions, the concept of using 
both of these two extreme $C R$ values is the main strategy in this study. It has been shown in the second preliminary experiment that using them in an adaptive switching manner controlled by the probabilities based on the success counts in the selection operator gives much better improvement than using them in a simple mixing manner with equal probability. Hence, this adaptive mixing strategy is employed in the DEASC algorithm. Note that the population size $N P=40$ is chosen as it can provide best results for all four representative test functions at both $D=10$ and $D=30$ with the smallest numbers of function evaluations.

In section 5.1, the performance comparison of DEASC and basic DE algorithms is carried out. Based on the value to reach $V T R=10^{-10}$, the comparison experiment finds the numbers of function evaluations required by each algorithm to reach the function values less than the $V T R$ for each test function. It has been shown that using the adaptive switching of low and high $C R$ values in combination with $F$ values in $[0.5,0.7]$ can enhance the performances of basic DE greatly and allows the use of a fixed small population size $(N P=40)$ for various and relatively high dimensional test functions. The DEASC algorithm can successfully solve all types of functions as expected, and its performances on high dimensional functions $(D=50,100)$ are outstanding.

The performance comparison of DEASC and some well-known adaptive DE variants is conducted in section 5.2. The comparison is based on various settings of maximum numbers of function evaluations allowed for each test function and at each dimension, as set in the original algorithms. The comparison experiments find the mean function values obtained by each algorithm for each test function. Despite its much simpler structure, the DEASC algorithm significantly outperforms all the compared methods. This result suggests a direction of designing an efficient adaptive DE variant by properly incorporating some useful recommendations from the studies on control parameters of DE.

In section 6, the DEASC algorithm is applied to solve some real-world problems of nonlinear systems of equations. These systems are highly nonlinear, multimodal, and nonseparable. It has been shown that our proposed algorithm can solve them very well and can also provide the solutions with high precisions.

\section{Conclusions}

In this research, an enhanced differential evolution algorithm called DEASC is presented for solving continuous optimization problems. The algorithm has a simple structure consisting of suitable population size, good choice of scaling factors, and the adaptive switching crossover strategy of using low or high crossover rates. The experimental results show that it remarkably enhances the performance of the basic DE algorithm and outperforms several well-known enhanced DE variants in the literature. Its performances are good and robust for all selected test functions of different types and difficulties, and some application problems of solving nonlinear systems. It also gives outstanding performance for high dimensional test functions. This suggests its use as a basic general-purpose method for global continuous optimization. Fur- 
ther study could investigate incorporating some special mutation strategies into the DEASC and applying the resulting algorithm to other application problems.

\section{Acknowledgements}

The authors thank Department of Mathematics, Faculty of Science, Khon Kaen University, for simulation equipment support.

\section{References}

[1] Al-Dabbagh R. D., Neri F., Idris N., Baba M. S., Algorithmic design issues in adaptive differential evolution schemes: Review and taxonomy, Swarm and Evolutionary Computation, 43, 2018 , 284-311.

[2] Boussaïd I., Lepagnot J., Siarry P., A survey on optimization metaheuristics, Information sciences, 237, 2013, 82-117.

[3] Brest J., Greiner S., Bokovi B., Mernik M., Žumer V., Self-adapting control parameters in differential evolution: A comparative study on numerical benchmark problems, IEEE Trans Evol Comput, 10, 6, 2006, 646-657.

[4] Das S., Konar A., Chakraborty U. K., Two improved differential evolution schemes for faster global search, in : Proceedings of the 2005 conference on genetic and evolutionary computation, 2005, 991-998.

[5] Das S., Mullick S.S., Suganthan P., Recent advances in differential evolution An updated survey, Swarm and Evolutionary Computation, 27, 2016, 1-30.

[6] Dennis J. E., Schnabel R. B., Numerical methods for unconstrained optimization and nonlinear equations, SIAM,Philadelphia, 1996.

[7] Gamperle R., Muller S. D., Koumoutsakos P., A parameter study for differential evolution, in: A. Gremla, N. E. Mastorakis (eds.), Advances in intelligent systems, fuzzy systems, evolutionary computation, WSEAS Press, Interlaken, 2002, 293-298.

[8] Hamm L., Brorsen B. W., Hagan M. T., Comparison of stochastic global optimization methods to estimate neural network weights, Neural Process Lett, 26, 2007, 145-158.

[9] Hirsch M. J., Pardalos P. M., Resende M. G. C., Solving systems of nonlinear equations with continuous GRASP, Nonlinear Analysis: Real World Applications, 10, 2009, 2000-2006.

[10] Lampinen J., Zelinka I., On stagnation of the differential evolution algorithm, in: R. Matouek, P. Omera (eds.), Proceedings of Mendel 2000, 6th international conference on soft computing, 2000, 76-83. 
[11] Leon M., Xiong N., Adapting differential evolution algorithms for continuous optimization via greedy adjustment of control parameters, Journal of artificial intelligence and soft computing research, 6, 2, 2016, 103-118.

[12] Li W., Li S., Chen Z., Zhong L., Ouyang C., Self-feedback differential evolution adapting to fitness landscape characteristics, Soft Comput, 23, 2019, 1151-1163.

[13] Mallipeddi R., Suganthan P. N., Empirical study on the effect of population size on differential evolution algorithm, in: Proceeding of the IEEE congress on evolutionary computation (CEC-2008), 2008, 3663-3670.

[14] Meintjes K., Morgan A., Chemical equilibrium systems as numerical test problems, ACM Transaction on Mathematical Software, 16, 1990, 143-151.

[15] Mezura-Montes E., Velzquez-Reyes J., Coello Coello C. A., A comparative study of differential evolution variants for global optimization, in: Proceedings of the 8th annual genetic and evolutionary computation conference (GECCO-2006), 2006, 485-492.

[16] Morgan A., Shapiro V., Box-bisection for solving second-degree systems and the problem of clustering, ACM Transaction on Mathematical Software, 13, 1987 , $152-167$.

[17] Nanda S. J., Panda G., A survey on nature inspired metaheuristic algorithms for partitional clustering, Swarm and Evolutionary Computation, 16, 2014, 1-18.

[18] Neri F., Tirronen V., Recent advances in differential evolution: A survey and experimental analysis, Artif Intell Rev, 33, 2010, 61-106.

[19] Oliveira H. A., Petraglia A., Solving nonlinear systems of functional equations with fuzzy adaptive simulated annealing, Applied Soft Computing, 13, 2013, 43494357.

[20] Ortega J. M., Rheinboldt W. C., Iterative solution of nonlinear equations inseveral variables, SIAM, Philadelphia, 2000.

[21] Pramanik S., Kinematic synthesis of a six-member mechanism for automotive steering, ASME Journal of Mechanical Design, 124, 2002, 642-645.

[22] Qin A.K., Huang V. L., Suganthan P. N., Differential evolution algorithm with strategy adaptation for global numerical optimization, IEEE Trans Evol Comput, 13, 2, 2009, 398-417.

[23] Ronkkonen J., Kukkonen S., Price K. V., Real parameter optimization with differential evolution, in : Proceedings of the IEEE congress evolutionary computation (CEC-2005) vol 1, IEEE Press, 2005, 506-513.

[24] Storn R., Differential evolution research-trends and open questions, in: U. K. Chakraborty (ed.), Advances in Differential Evolution, Springer, Berlin, 2008, 1-31. 
[25] Storn R., Price K., Differential evolution - A simple and efficient adaptive scheme for global optimization over continuous spaces, Technical Report TR-95012, ICSI, 1995.

[26] Storn R., Price K., Differential evolution: A simple and efficient heuristic for global optimization over continuous spaces, J Glob Optim, 11, 4, 1997, 341-359.

[27] Venter G., Review of optimization techniques, in: R. Blockley, S. Wei (eds.), Encyclopedia of aerospace engineering, Wiley and Sons, 2010, doi: 10.1002/9780470686652.eae495.

[28] Verschelde J., Verlinden P., Cools R., Homotopies exploiting newton polytopes for solving sparse polynomial systems, SIAM J. Numer. Anal, 31, 1994, 915-930.

[29] Wongpen J., Wetweerapong J., Puphasuk P., Finding a maximum clique in social networks using a modified differential evolution algorithm, WSEAS Transactions on Systems and Control, 14, 2019 , 333-342.

[30] Zhang J., Sanderson A. C., JADE: adaptive differential evolution with optional external archive, IEEE Trans Evol Comput, 13, 5, 2009, 945-958.

[31] Zielinski K., Weitkemper P., Laur R., Kammeyer K., Parameter study for differential evolution using a power allocation problem including interference cancellation, in: IEEE congress on evolutionary computation (CEC-2006), 2006, 1857-1864.

Received 7.02.2020, Accepted 16.05.2020 